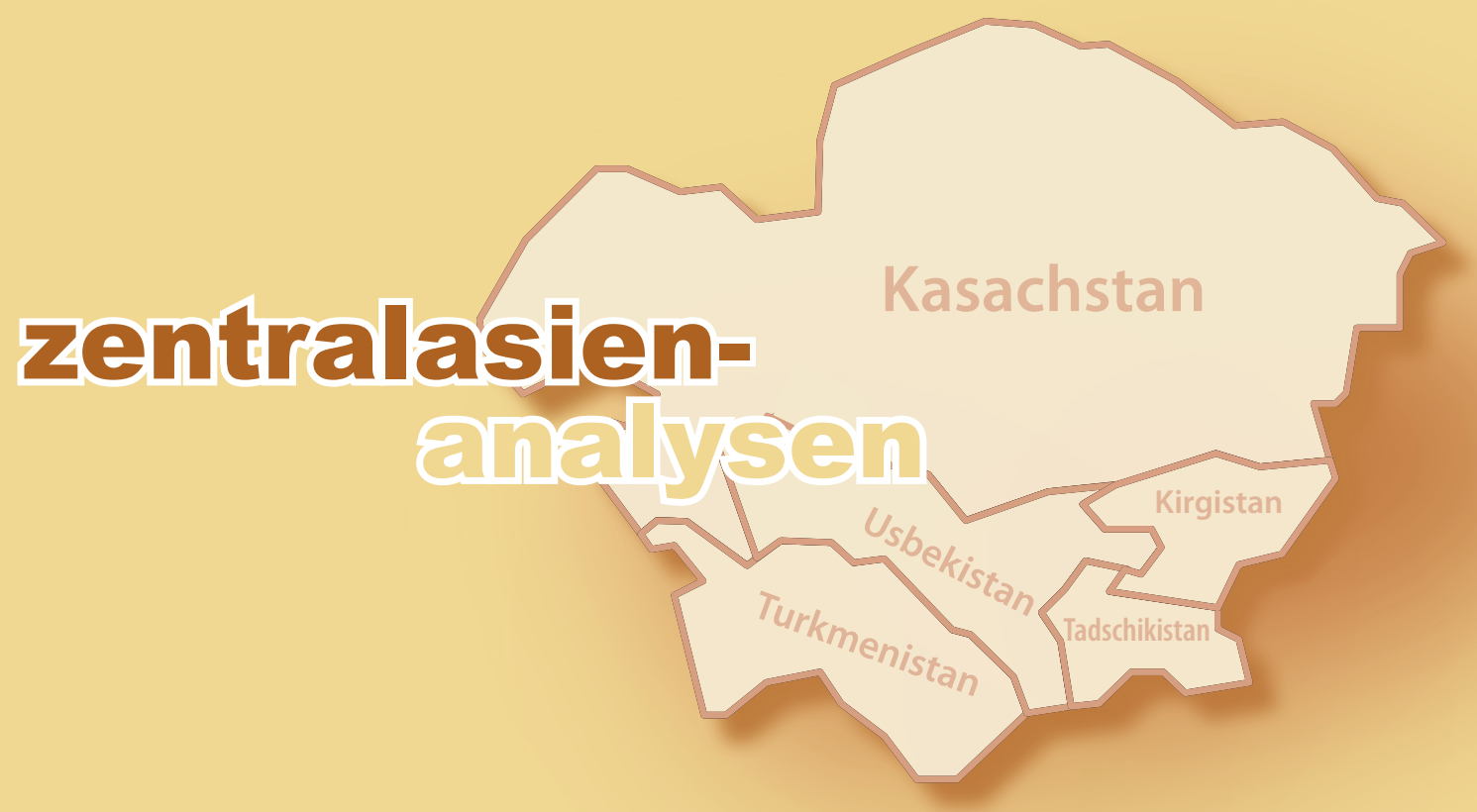

\title{
CHINESISCHE ZENTRALASIENFORSCHUNG
}

\section{ANALYSE}

Zentralasien mit den Augen Chinas.

Wissensproduktion, akademische Wahrnehmung und Folgen für die Politik

Von Assel Bitabarova, Sapporo

- TABELLE ZUM TEXT

Übersicht über die wichtigsten Universitäts- und Forschungseinrichtungen für

Zentralasienstudien in China

CHRONIK

26. März - 22. April 2016

Kasachstan 9

$\begin{array}{lr}\text { Kirgistan } & 10\end{array}$

$\begin{array}{lr}\text { Tadschikistan } & 13\end{array}$

Turkmenistan $\quad 15$

$\begin{array}{ll}\text { Usbekistan } & 16\end{array}$ 


\section{Zentralasien mit den Augen Chinas. Wissensproduktion, akademische Wahrnehmung und Folgen für die Politik}

Von Assel Bitabarova, Sapporo

\section{Zusammenfassung}

Die Unabhängigkeit der fünf zentralasiatischen Staaten infolge des plötzlichen Zusammenbruchs der Sowjetunion im Jahr 1991 hat man in China genauso wenig vorhergesehen wie in der übrigen Welt. Zu jener Zeit hatten die politischen Verantwortlichen in Beijing weder eine klare Vorstellung von ihren neuen Nachbarn noch waren sie darauf vorbereitet, in dieser neuen politischen Situation begründete Entscheidungen zu treffen. Heute, ein Vierteljahrhundert später, ist China zu einem der wichtigsten Partner der zentralasiatischen Staaten geworden. Auf der außenpolitischen Agenda Chinas hat die Region an Profil gewonnen. Dies verdeutlicht auch die Tatsache, dass Staats- und Parteichef Xi Jinping im September 2013 Astana als Schauplatz für die Verkündung der Initiative "Silk Road Economic Belt« (Teil der Strategie »One Belt One Road «) gewählt hat, die seither zum Eckpfeiler seiner neuen Außenpolitik geworden ist. Parallel zur wachsenden Aufmerksamkeit, die Politiker in Beijing der Region widmen, haben auch akademische Kreise in China zunehmendes Interesse an Zentralasien gezeigt. Der folgende Beitrag gibt auf der Basis chinesischer Quellen und aktueller Interviews mit chinesischen Forschern einen Überblick über die Entwicklung der Zentralasienforschung in China und ihre Verbindung mit der Politik Beijings.

\section{Beginn der chinesischen Zentralasienforschung}

Die Zentralasienforschung in China entstand während der Sowjetzeit innerhalb des größeren Rahmens der Sowjetunion- und Osteuropastudien, die allerdings bis Ende der 1970er Jahre aufgrund der verheerenden Auswirkungen der Kulturrevolution kaum Fortschritte machten. Die Institutionalisierung der chinesischen Zentralasienforschung begann erst mit der Einrichtung von Forschungsabteilungen an der Universität Xinjiang im Jahre 1979 und an der Akademie für Sozialwissenschaften (XASS) in Xinjiang 1980. Kurz darauf, im Jahr 1991, wurde die Welt Zeuge der Auflösung der Sowjetunion, und die früheren Sowjetrepubliken wurden souveräne Staaten.

In den ersten Jahren nach der Unabhängigkeit der zentralasiatischen Staaten hatte die chinesische Führung keine ausgearbeitete Vision oder klare Strategie gegenüber der Region, sondern war lediglich darum bemüht, mögliche negative Auswirkungen des Zusammenbruchs der Sowjetunion zu verhindern und mit dem Auftauchen der neuen Nachbarn verbundene Unsicherheiten zu minimieren. Die Hauptaufgaben bestanden also darin, Lösungen für zentrale Sicherheitsprobleme zu finden, nämlich: (1) die Sicherheit entlang der über $3.000 \mathrm{~km}$ langen chinesisch-zentralasiatischen Grenze durch Entmilitarisierung und die Beilegung von Grenzstreitigkeiten mit Kasachstan, Kirgistan und Tadschikistan aufrecht zu erhalten; (2) die Ausbreitung separatistischer Bestrebungen in der Autonomen Uighurischen Region Xinjiang (XUAR) zu verhindern, denn Funktionäre in Beijing fürchteten, dass ein stärkeres nationa- les Selbstbewusstsein der zentralasiatischen Nachbarn nach Erlangung ihrer Souveränität auch nationalistische Bestrebungen bei den Uighuren auslösen könnte. Die Bewahrung von Stabilität und innerer Sicherheit in Xinjiang war entsprechend auch Schwerpunkt der chinesischen Zentralasienforschung jener Jahre.

Da der Staat auf Unterstützung von Seiten der Wissenschaft angewiesen war, hatten einige der damaligen Forschungsarbeiten entscheidenden Einfluss auf die Formulierung und Ausgestaltung der Politik Beijings. So konzentrierte sich zum Beispiel Xing Guangcheng, einer der einflussreichsten chinesischen Russland- und Zentralasienexperten, in den 1990er Jahren stark auf politikorientierte Forschung sowie auf geopolitische Analysen und Sicherheitsfragen in Zentralasien. Seine Monographien über Chinas Beziehungen zu den neuen unabhängigen Staaten der Region gehören zu den wenigen wissenschaftlichen Arbeiten über Zentralasien, die in China in jener Periode veröffentlicht wurden. Später, von 2003 bis 2009, fungierte er als Direktor des Instituts für Russland, Osteuropa und Zentralasien an der Chinesischen Akademie für Sozialwissenschaften (CASS), die zu den führenden Think Tanks des Landes gehört und eng mit der chinesischen Regierung kooperiert. In diesem Zusammenhang muss man auch die Gründung des Instituts für Zentralasien- und Xinjiangstudien an der Universität Lanzhou erwähnen (später wurde es in Institut für Zentralasienstudien [ICAS] umbenannt), denn es ist inzwischen zu einem zentralen Forschungsstandort für Zentralasien innerhalb des chinesischen Universitätssystems (gaoxiao) geworden. Insgesamt gab es im ersten Jahrzehnt nach der Entstehung der neuen Staa- 
ten Zentralasiens aber nur wenig wissenschaftliche Forschung über diese Region und ihr Niveau war nicht hoch.

\section{Aufbruch ins neue Jahrtausend}

Seit Beginn des neuen Jahrtausends, als Chinas Aufstieg immer sichtbarer wurde, begann die Führung in Beijing, den Forschungsinstituten sehr viel mehr Beachtung zu schenken. Sie bildete innerhalb des Universitätssystems neue Forschungsschwerpunkte in den Geistes- und Sozialwissenschaften und stellte auf dem Weg über die Nationale Sozialwissenschaftliche Stiftung mehr Mittel für Forschung zur Verfügung. Die Gründung neuer Forschungseinrichtungen mit Schwerpunkt Zentralasien erfolgte sowohl innerhalb regierungsnaher Think Tanks als auch an mehreren Universitäten. Beispielsweise wurde 2004 an der Shaanxi Normal University in Xi'an das Institut für Zentralasienstudien gegründet; 2005 das Zentrum für Russland- und Zentralasienforschung an der Fudan-Universität in Shanghai. Die Forschungsgruppe für Eurasienstudien am Chinesischen Institut für Internationale Studien (CIIS) in Beijing wurde zur Abteilung für Europa- und Zentralasienforschung aufgewertet und vergrößert.

Wie sind diese Institutionen nun charakterisiert? Zum einen ist die Mehrzahl innerhalb breiterer, traditionell auf den früheren sowjetischen Raum konzentrierter Forschungsstrukturen angesiedelt, auch wenn es einige relativ gut etablierte, gesonderte Forschungsinstitutionen gibt, deren Fokus in erster Linie auf Zentralasien liegt - wie z. B. das ICAS an der Universität Lanzhou oder die Abteilung für Zentralasienstudien an der XASS. Ein anderes auffälliges Merkmal betrifft die Zuständigkeitsverteilung der Wissensproduktion über Zentralasien: Während die Forschung in Beijing und Shanghai in erster Linie an Politik und Strategieentwicklung orientiert ist, umfasst die Zentralasienforschung in Xinjiang (mehrere Forschungsinstitutionen wie die XASS und die Xinjiang University) ein wesentlich breiteres Spektrum an Forschungsthemen und -gebieten und hat insbesondere geisteswissenschaftliche Expertise hervorgebracht. Das ICAS an der Lanzhou University bietet umfassende Regionalforschung an, in den vergangenen Jahren hat sich ein Fokus auf Sicherheitsfragen herausgebildet. Das wichtigste Forschungszentrum in Xi'an, die Shaanxi Normal University, hat sich vor allem auf Geschichte und Erforschung ethnischer Minderheiten spezialisiert. Die in Beijing-basierte Zentralasien-Forschung wird damit als besonders einflussreich für den politische Entscheidungsprozess angesehen, während Xinjiang seine günstige Position zur Rekrutierung von Vertretern nationaler Minderheiten, etwa Kasachen und Kirgisen, die die Titularsprache der westlichen Nachbarstaaten beherrschen, nutzt.
Hinzu kommt, dass in den letzten Jahren an den chinesischen Universitäten ein auffälliger Trend zur Gründung von sog. Zentralasien-Forschungsinstituten zu verzeichnen ist - so haben zum Beispiel allein in Xi'an mehrere Universitäten neue Institute für Zentralasienstudien geschaffen. Ihr Hauptzweck ist jedoch nicht, die akademische Forschung zu fördern, sondern internationale Studierende aus den Ländern Zentralasiens zu gewinnen.

Einer der auffallendsten Forschungstrends im Zusammenhang mit Chinas Zentralasienpolitik ist die Ausweitung der Forschung zur Shanghai Organisation für Zusammenarbeit (SCO). Wie bekannt, handelt es sich bei der SCO um eine Organisation zur regionalen Zusammenarbeit, die im Jahre 2001 von China, Russland und den vier zentralasiatischen Staaten Kasachstan, Kirgistan, Tadschikistan und Usbekistan offiziell gegründet wurde. Seit ihrer Gründung haben sich dank der Unterstützung der Regierung die akademische Beschäftigung mit dieser Organisation und der Kenntnisstand über ihre politische Entwicklung extrem schnell ausgeweitet. Forschung zur SCO wird in China in Dutzenden von Organisationsstrukturen im ganzen Land betrieben, besonders stark ist sie an den Forschungseinrichtungen in Beijing und Shanghai konzentriert. Fast an jedem großen Think Tank in diesen Städten gibt es SCO-Experten. Hierzu zählen zum Beispiel das Chinesische Zentrum für SCO-Studien an der CIIS (Beijing), das Zentrum für SCO-Studien an der CASS (Beijing) sowie Zentren gleichen Namens an der Akademie für Sozialwissenschaften (SASS) und an der Fudan-Universität in Shanghai etc. So sind etwa Regierungsvertreter, Forscher bzw. Experten von Universitäten und Think Tanks Mitglieder des Chinesischen Zentrums für SCO-Studien an der CIIS; dessen jährliche Treffen stellen eine Plattform für den Austausch zwischen Regierung und akademischer Welt dar.

Es ist nicht überraschend, dass die Entwicklung der Forschung zur sog. One Belt - One Road (OBOR)-Initiative nach demselben Muster verläuft. Die OBORInitiative des chinesischen Präsidenten Xi Jinping verknüpft zwei außenwirtschaftliche Konzepte: »Silk Road Economic Belt«, als Verbindung Chinas mit Europa auf dem Landweg durch Zentralasien, sowie die »Maritime Silk Road « [die chinesische Häfen mit dem Nahen und Mittleren Osten sowie der afrikanischen Ostküste verbinden soll] (Anm. d. Redaktion). Allein in den letzten beiden Jahren sind Dutzende von neuen Forschungseinrichtungen zu diesem groß angelegten Vorhaben gegründet worden. Die OBOR-Strategie scheint zum heißesten Forschungsthema geworden zu sein - oder zum lukrativsten, wie einige chinesische Experten scherzen.

Obwohl das Wissen über Zentralasien in China also ständig wächst, befindet es sich immer noch in 
einem frühen Entwicklungsstadium. Chinesische Wissenschaftler weisen darauf hin, dass die Entwicklung der Zentralasienforschung durch einige Hemmnisse behindert wird. Dazu gehören das relativ niedrige akademische Niveau, unterentwickelte Forschungsmethoden sowie ein Mangel an Fachleuten [mit entsprechenden Fremdsprachenkenntnissen] (Anm. d. Redaktion), die tatsächlich Zentralasienstudien als Hauptfach belegt haben. Denn die Mehrzahl der chinesischen Experten, die sich mit Zentralasien befassen - besonders in Beijing und Shanghai -, hat ihre Ausbildung ursprünglich im Bereich der Russlandstudien erhalten. Dies hat dazu geführt, dass die Russlandforschung generell als »Hauptforschungsfeld " (zhuye) angesehen wird, die Zentralasienforschung dagegen als »Nebensache» (fuye) .

\section{Alte und neue Verbindungen}

In frühen chinesischen Quellen [insbesondere der HanPeriode] (Anm. d. Redaktion) liegen die heutigen zentralasiatischen Staaten innerhalb der Region, die [bis zum Ende des 19. Jahrhunderts] (Anm. d. Redaktion) "xiyu«, also "Westliche Region(en)« genannt wurde, obwohl kein zeitgenössischer Forscher die Grenzen dieser Region exakt definieren könnte. Fragt man chinesische Zentralasienexperten heute nach ihrem grundsätzlichen Bild von Zentralasien, so wird jedoch fast jeder auf xiyu verweisen; ein Konzept, das gewöhnlich mit romantischen Vorstellungen über die historischen Austauschbeziehungen zwischen den Völkern der Westlichen Region und dem chinesischen Kernland assoziiert wird.

Ausgehend von Präsident Xis Seidenstraßen-Initiative können wir derzeit einen anhaltenden Forschungsboom beobachten, der sich mit der alten Seidenstraße und ihrer modernen Version befasst. Einige chinesische Wissenschaftlicher weisen darauf hin, dass die Rolle Zentralasiens als Brücke zwischen Osten und Westen durch die nördliche Ausrichtung der dort unter russischer und sowjetischer Herrschaft gebauten Verkehrsinfrastruktur in den Hintergrund gedrängt wurde. Heute fordert Zentralasien seine historische Rolle jedoch zurück, davon zeugen ihrer Ansicht nach die gemeinsamen zentralasiatisch-chinesischen (oft von China finanzierten) Infrastrukturprojekte, die nicht nur die regionale Integration fördern wollen, sondern auch den Handel zwischen den Großregionen.

Wenn man über historische Bindungen bzw. kulturelle Verwandtschaft zwischen den Völkern Chinas und seinen zentralasiatischen Nachbarn spricht, bezieht sich das gewöhnlich auf die ethnischen Minderheiten (minzu), die diesseits und jenseits der Grenzen zwischen China und Zentralasien leben und eine gemeinsame Sprache, Kultur und Religion haben, wie Kasachen,
Kirgisen, Dunganen, Uighuren, Usbeken und Pamiris [Letztere in China zusammenfassend Tadschiken genannt] (Anm. d. Redaktion) etc. Zu diesen ethnischen Gruppen sind in China beachtliche Forschungen durchgeführt worden, hauptsächlich von Wissenschaftlern aus dem westlichen Teil des Landes. Dennoch betonen die meisten Experten, dass die breite Öffentlichkeit in China - mit Ausnahme der Bevölkerung in den Grenzregionen und der Regionen mit bedeutenden verwandten ethnischen Minderheiten - nur geringe Kenntnisse über Zentralasien hat. Sie sagen außerdem, dass dies auf den ersten Blick zwar paradox erscheine, aber doch einleuchtend sei, denn Xinjiang wurde bis vor wenigen Jahren in der Öffentlichkeit als natürliche Pufferzone zwischen dem chinesischen Kernland und xiyu angesehen. Wenn Xinjiang eine riesige Pufferzone zwischen China und Zentralasien bildet, dann stellt Zentralasien eine Pufferzone zwischen China und Russland dar.

Die Beziehungen zu Russland gehören zudem zu den Schlüsselfaktoren, die die Zentralasienpolitik der chinesischen Führung bestimmen. Der offizielle chinesische Diskurs, dass "China die sspeziellen Interessen Russlands in Zentralasien anerkennt", wird im Wesentlichen von den Wissenschaftlern geteilt. Chinesische Forscher sind sich der skeptischen Haltung Russlands gegenüber der chinesischen Zentralasienpolitik bewusst und erwägen gründlich, wie Russland auf etwaige politische Schritte und Maßnahmen Chinas in der Region reagieren könnte, indem sie die Präferenzen aller beteiligten Akteure einbeziehen. Sie erkennen außerdem die Tatsache an, dass Russland nicht nur die Position eines "traditionellen Verbündeten Zentralasiens« oder "Sicherheitsgaranten« einnimmt, sondern auch über starken kulturellen Einfluss in der Region verfügt, während China von einem ähnlich bedeutenden Status in der Region (noch) weit entfernt ist. Ein renommierter chinesischer Experten formulierte das gegenüber der Autorin folgendermaßen: »Die zentralasiatischen Staaten und Russland können Meinungsverschiedenheiten haben und miteinander diskutieren, aber von uns aus betrachtet ist das ein Familienstreit. Sie gehören alle zu einer Familie.« Andrerseits sind die Beziehungen der zentralasiatischen Länder zu ihrem früheren Zentrum Russland asymmetrisch, der "russische Bruder" handelt oft als "älterer Bruder" und wird gewöhnlich so wahrgenommen. In dieser Hinsicht begrüßen die zentralasiatischen Führungen die Präsenz Chinas als ein Gegengewicht zu Russland.

\section{Die Bedeutung der SCO}

Unter dem Aspekt des Machtgleichgewichts hat die SCO für alle Mitglieder eine wichtige Funktion. Chinesische Experten unterstreichen die Bedeutung der SCO und 
insbesondere die Bedeutung der Mitgliedschaft Chinas für die zentralasiatischen Staaten. Diese sind bestrebt, Russlands starkem Einfluss etwas entgegen zu setzen. Außerdem sind chinesische Wissenschaftler der Ansicht, dass die Organisation eine gute Plattform für eine vertiefte wechselseitige Wahrnehmung und Kommunikation darstellt und man sich in ihrem Rahmen mit den Interessen und Problemen der anderen Mitgliedsstaaten besser vertraut machen kann.

Seit der Gründung der SCO haben sich chinesische Wissenschaftler und Experten in zahlreichen Forschungsprojekten mit dieser Organisation befasst. Zwar schwanken ihre Bewertungen, was ihre Wirkungsmächtigkeit und ihren Erfolg angeht, zwischen "gut" und "wenig effektiv«, doch niemand zweifelt an ihrer Bedeutung. In diesem Zusammenhang ist die Sicherheitsfrage immer von eminenter Bedeutung für die Führung in Beijing gewesen. Dahinter steckt das Ziel, Sicherheit und eine stabile Entwicklung in den nordwestlichen Grenzregionen, vor allem in Xinjiang, zu gewährleisten. Die Kooperation mit den westlichen Nachbarländern bei der Bekämpfung der »drei Übel« des Terrorismus, des Separatismus und des Extremismus sowie auch des Drogenhandels und der internationalen Kriminalität ist gemeinsames Ziel aller Mitglieder. Zweitens gehören die Grundprinzipien der Organisation - wechselseitiger Respekt für die Souveränität und territoriale Integrität der Mitgliedsstaaten und Nichteinmischung in ihre inneren Angelegenheiten - ebenfalls zu den fundamentalen Grundsätzen der chinesischen Diplomatie. Die Mitgliedsstaaten der SCO unterstützen Beijings "Ein China-Prinzip« ebenso wie seine politische Strategien zur Lösung der "uighurischen Frage«.

Was das Prinzip der Nichteinmischung in innere Angelegenheiten betrifft, so kann man zwei Hauptrichtungen ausmachen. Die Mehrheitsmeinung innerhalb der SCO unterstützt das Prinzip der Nichteinmischung und lehnt Interventionen ab. Angesichts der Reaktion der SCO auf die seit Jahren bestehenden Spannungen zwischen Uighuren und Han in Xinjiang ist dies offensichtlich. Andererseits vertreten einige Experten die Meinung, dass die SCO sich aktiv für die Sicherheit in der Region einsetzen und, wenn es erforderlich ist, auf innere Destabilisierungsprozesse oder Krisen unpolitischer Natur reagieren sollte. Als negatives Beispiel haben etwa die ethnischen Zusammenstöße im kirgisischen Osch im Jahr 2010 den geringen Zusammenhalt innerhalb der SCO und ihre geringen Einfluss augenscheinlich werden lassen, denn die Organisation war nicht in der Lage, konkrete Mechanismen zur Konfliktvermittlung anzubieten.
Hinzu kommt, dass in China, aber auch anderswo, allgemein zugegeben wird, dass die SCO bislang nicht besonders erfolgreich dabei war, die wirtschaftliche Zusammenarbeit zwischen ihren Mitgliedsstaaten zu intensivieren. Wichtige wirtschaftliche Kooperationen und Investitionsprojekte in großem Maßstab wurden bislang hauptsächlich im Rahmen bilateraler Beziehungen verwirklicht. Dennoch glauben chinesische Experten, dass die SCO ein großes Potential für die Entfaltung multilateraler wirtschaftlicher Zusammenarbeit besitzt, denn eine nachhaltige Wirtschaftsentwicklung in der Region ist die wichtigste Grundlage für die Garantie regionaler Sicherheit.

\section{Fazit: Verschränkung von Wissenschaft und Politik}

Die akademische Forschung zu Zentralasien scheint in China für politische Entscheidungsprozesse relevant zu sein und der wissenschaftliche Diskurs hat über direkte oder indirekte Informationskanäle Einfluss auf die politischen Strategien der Regierung. Beispielsweise werden Forschern gelegentlich Forschungsprojekte übertragen, welche die Regierung in Auftrag gegeben hat, um Empfehlungen für politische Maßnahmen auszuarbeiten. Ein anderes Beispiel ist die Berufung von Experten als Politikberater für Behörden. Wie oben skizziert, hat die chinesische Regierung für die Entwicklung und Institutionalisierung der Wissensproduktion in diesem Bereich umfangreiche Unterstützung bereitgestellt. Man kann schlussfolgern, dass die Bedeutung der Region Zentralasien für China zunehmend anerkannt wird. Allerdings ist der erwähnte Mangel an qualifizierten, auf Zentralasien spezialisierten Wissenschaftlern nach wie vor ausgeprägt. Und dieses Problem hat zwei, auf den ersten Blick paradoxe, Folgen. Einerseits sind »rares Fachwissen und Regionalkenntnisse « besonders wertvoll, und es ist denkbar, dass sie deshalb umso größeren Einfluss auf politische Entscheidungen haben. Andererseits weisen viele chinesische Wissenschaftler darauf hin, dass der Mangel an kompetenten Experten nicht nur die Entwicklung der Regionalwissenschaft behindert, sondern auch insbesondere die Qualität der politikrelevanten Forschung. Von daher bleibt der Ausbau der Zentralasienforschung und der entsprechenden wissenschaftlichen Ausbildung eine wichtige Aufgabe für das heutige China.

Aus dem Englischen von Brigitte Heuer

Informationen über die Autorin und Lesetipps finden Sie auf der nächsten Setie. 
Über die Autorin:

Assel Bitabarova stammt aus Astana, Kasachstan, und promoviert gegenwärtig im dritten Studienjahr am Graduiertenkolleg der Universität von Hokkaido im japanischen Sapporo im Fach Zentralasienstudien. Sie hat im Rahmen von Aufenthalten in Beijing und Shanghai im September 2013 sowie in Xi'an, Lanzhou und Urumtschi im Januar 2016 zur Rolle Zentralasiens in den chinesischen Sozialwissenschaften geforscht. Zu ihren wissenschaftlichen Interessen gehören Internationale Beziehungen und Sicherheitsfragen in Zentralasien, die Beziehungen zwischen China und Zentralasien sowie Chinas internationale Entwicklungszusammenarbeit.

\section{Lesetipps:}

- Raffaello Pantucci, Central Asia: The view from China, European Union Institute for Security Studies, Alert 3, January 2015, = <http://www.iss.europa.eu/uploads/media/Alert_3_Central_Asia_China.pdf $>$

- China’s Central Asia Problem, International Crisis Group (ICG) Asia Report No. 244, 27 February $2013=<$ http:// www.crisisgroup.org/-/media/Files/asia/north-east-asia/244-chinas-central-asia-problem.pdf>

- Nadine Godehardt, Chinas «neue« Seidenstraßeninitiative. Regionale Nachbarschaft als Kern der chinesischen Außenpolitik unter Xi Jinping, SWP-Studien 2014/S 09, Juni 2014, = <http://www.swp-berlin.org/fileadmin/con tents/products/studien/2014_S09_gdh.pdf>

- Nadine Godehardt, The Chinese Constitution of Central Asia. Regions and Intertwined Actors in International Relations, Houndmills/Basingstoke, New York 2014, 232 S.

\section{Übersicht über die wichtigsten Universitäts- und Forschungseinrichtungen für Zentralasienstudien in China}

\begin{tabular}{|c|c|c|}
\hline Ort & Name & Kurzbeschreibung \\
\hline \multirow[t]{2}{*}{ Beijing } & $\begin{array}{l}\text { Chinese Academy of Social } \\
\text { Sciences (CASS), Institute } \\
\text { for Russian, East European } \\
\text { and Central Asian Studies } \\
\text { (IREECAS), Department } \\
\text { for Central Asian Studies } \\
\text { (DCAS) }\end{array}$ & $\begin{array}{l}\text { Das Institute for Russian, East European and Central Asian Studies } \\
\text { an der CASS wird zu den führenden chinesischen Forschungs- } \\
\text { einrichtungen für den Bereich der ehemaligen Sowjetrepubliken } \\
\text { gezählt. Die Abteilung für Zentralasienstudien wurde } 1992 \text { gegrün- } \\
\text { det, gegenwärtig arbeiten dort sechs Forscher mit breiter Expertise. } \\
\text { Ein besonderer Schwerpunkt liegt auf aktuellen politischen Fragen. } \\
\text { CASS war die erste akademische Institution im Lande, an der Dis- } \\
\text { sertationen zum Thema Zentralasien abgeschlossen wurden. Seit } \\
1981 \text { gab die CASS die wissenschaftliche Zeitschrift Soviet and East } \\
\text { European Studies heraus, die später in Russian, Central Asian \& } \\
\text { East European Studies (informelle Übersetzung des Titels, erscheint } \\
\text { nur chinesisch, Anm. der Red.) umbenannt wurde. } \\
\text { Website: <http://euroasia.cssn.cn/zyyjw/> (nur chinesisch) }\end{array}$ \\
\hline & $\begin{array}{l}\text { China Institute of In- } \\
\text { ternational Studies (CIIS), } \\
\text { Department for European } \\
\text { and Central Asian Studies } \\
\text { (DECAS) }\end{array}$ & $\begin{array}{l}\text { Beim CIIS handelt es sich um einen dem Außenministerium an- } \\
\text { geschlossenen Think Tank, der unmittelbar an Politikanalysen über } \\
\text { ein breites Spektrum außenpolitischer Fragen mitwirkt. DECAS } \\
\text { führt Forschungen und Analysen über die gegenwärtige politische } \\
\text { Lage, die Außenpolitik und regionale Organisationen im postsowje- } \\
\text { tischen Raum zum Zweck der Politikberatung durch. } \\
\text { Website: <http://www.ciis.org.cn/english/2010-11/04/con } \\
\text { tent_3813484.htm> (chinesisch und kurze englische Version) }\end{array}$ \\
\hline
\end{tabular}




\section{Übersicht über die wichtigsten Universitäts- und Forschungseinrichtungen für Zentralasienstudien in China (Fortsetzung)}

\begin{tabular}{|c|c|c|}
\hline Ort & Name & Kurzbeschreibung \\
\hline \multirow[t]{2}{*}{ Beijing } & $\begin{array}{l}\text { China Institute of Contem- } \\
\text { porary International Rela- } \\
\text { tions (CICIR), Department } \\
\text { for Central Asian Studies } \\
\text { (DCAS) }\end{array}$ & $\begin{array}{l}\text { Die Forschungsgruppe Zentralasien des CICIR umfasst nur wenige } \\
\text { Personen, doch diese verfügen über breites Fachwissen mit einem } \\
\text { besonderen Schwerpunkt auf politik- und strategieorientierter } \\
\text { Forschung, wie z. B. die Politik der einzelnen zentralasiatischen } \\
\text { Staaten, Außenpolitik, bilaterale und multilaterale Wirtschafts- } \\
\text { beziehungen sowie Sicherheitsfragen. } \\
\text { Website: <http://www.cicir.ac.cn/chinese/Organ_58.html> (nur } \\
\text { chinesisch) }\end{array}$ \\
\hline & $\begin{array}{l}\text { Renmin University of China } \\
\text { (RUC), Institute for Russian, } \\
\text { East European and Central } \\
\text { Asian Studies (IREECA) }\end{array}$ & $\begin{array}{l}\text { Das Institut wurde } 1964 \text { als Institut für Sowjet- und Osteuropa- } \\
\text { studien gegründet und nach dem Zusammenbruch der Sowjetunion } \\
\text { umbenannt. Der Schwerpunkt der Forschung liegt hauptsächlich } \\
\text { auf Ökonomie und Politischen Wissenschaften. } \\
\text { Website der RUC: <http://www.ruc.edu.cn> (chinesisch und eng- } \\
\text { lisch = international version) }\end{array}$ \\
\hline \multirow{4}{*}{ Shanghai } & $\begin{array}{l}\text { Shanghai Academy of Social } \\
\text { Sciences (SASS), Institute } \\
\text { for Eurasian Studies (IES), } \\
\text { Department for Russia } \\
\text { and Central Asian Studies } \\
\text { (DRCAS) }\end{array}$ & $\begin{array}{l}\text { Dieses Institut wurde } 1981 \text { unter dem Namen Institut für Sowjet- } \\
\text { und Osteuropastudien gegründet und } 1992 \text { in Institut für Eurasien- } \\
\text { studien umbenannt. Es hat gegenwärtig einen Personalbestand von } \\
29 \text { Mitarbeitern und umfasst mehrere Forschungsgruppen, darunter } \\
\text { das DRCAS. Zu den Hauptuntersuchungsgebieten gehören Politik, } \\
\text { Wirtschaft, nicht-traditionelle Sicherheitsrisiken, Kultur, Beziehun- } \\
\text { gen Zentralasiens mit China u. a. } \\
\text { Website des SASS: < http://www.sass.org.cn/> (chinesisch und englisch) }\end{array}$ \\
\hline & $\begin{array}{l}\text { Shanghai Institute for Inter- } \\
\text { national Studies (SIIS), Cen- } \\
\text { tre for Russian and Central } \\
\text { Asian Studies (CRCAS) }\end{array}$ & $\begin{array}{l}\text { Das } 1960 \text { gegründete SIIIS gehört zu den einflussreichsten Think } \\
\text { Tanks Chinas. Es widmet sich besonders der Analyse von interna- } \\
\text { tionaler Politik, Wirtschaft, Sicherheitsstrategie und Außenbezie- } \\
\text { hungen Chinas. Zum Team des CRCAS gehören sechs Forscher, } \\
\text { deren Expertise sich auf die chinesisch-russischen und chinesisch- } \\
\text { zentralasiatischen Beziehungen sowie Untersuchungen zur SCO } \\
\text { konzentriert, außerdem beschäftigen sie sich mit Innenpolitik, } \\
\text { Sicherheit, Wirtschaft und den Außenbeziehungen Russlands und } \\
\text { der zentralasiatischen Staaten. } \\
\text { Website: <http://en.siis.org.cn/index.php?m=content\&c=index\&a= } \\
\text { show\&catid=20\&id=6> (chinesisch und englisch) }\end{array}$ \\
\hline & $\begin{array}{l}\text { East China Normal Uni- } \\
\text { versity (ECNU), Centre for } \\
\text { Russian Studies (CRS) }\end{array}$ & $\begin{array}{l}\text { Das } 1999 \text { gegründete Zentrum ist heutzutage eines der wichtigsten } \\
\text { nationalen Forschungsinstitute für Geistes- und Sozialwissenschaf- } \\
\text { ten innerhalb des chinesischen Universitätssystems. CRS befasst } \\
\text { sich mit dem postsowjetischen Raum einschließlich Zentralasien. } \\
\text { In den letzten zehn Jahren wurden hier } 44 \text { Forschungsprojekte } \\
\text { durchgeführt. ECNU gehört zu jenen chinesischen Universitäten, } \\
\text { an denen zahlreiche Doktorarbeiten im Bereich Zentralasienstudien } \\
\text { abgeschlossen wurden. } \\
\text { Website: <http://rus.ecnu.edu.cn/il311.htm> (neben chinesisch auch } \\
\text { englisch und russisch) }\end{array}$ \\
\hline & $\begin{array}{l}\text { Fudan University, Centre for } \\
\text { Russian and Central Asian } \\
\text { Studies (CRCAS) }\end{array}$ & $\begin{array}{l}\text { Das Zentrum wurde } 2005 \text { an der Universität Fudan ins Leben } \\
\text { gerufen. Zuvor existierte hier ein Zentrum für Russland- und } \\
\text { Osteuropastudien. Zu den Hauptforschungsgebieten gehören: } \\
\text { Beziehungen zwischen Russland und den zentralasiatischen Staaten, } \\
\text { Chinas Beziehungen zu Russland und Zentralasien, Untersuchun- } \\
\text { gen zur SCO. } \\
\text { Website: <http://www.iis.fudan.edu.cn/6765/list.htm> (nur chinesisch) }\end{array}$ \\
\hline
\end{tabular}




\section{Übersicht über die wichtigsten Universitäts- und Forschungseinrichtungen für Zentralasienstudien in China (Fortsetzung)}

\begin{tabular}{|c|c|c|}
\hline Ort & Name & Kurzbeschreibung \\
\hline \multirow[b]{2}{*}{ Xinjiang } & $\begin{array}{l}\text { Academy of Social Sciences } \\
\text { Xinjiang (XASS), Institute } \\
\text { for Central Asian Studies } \\
\text { (ICAS) }\end{array}$ & $\begin{array}{l}\text { Das Institute for Central Asian Studies an der XASS (gegründet } \\
\text { 1980) zählt zu den ältesten und einflussreichsten Think Tanks } \\
\text { des Landes und führt umfangreiche Forschungen zu Zentralasien } \\
\text { durch. Zu den Forschungsgebieten gehören Politik, Sicherheits- } \\
\text { fragen und Wirtschaft in Zentralasien und den angrenzenden } \\
\text { Regionen, Beziehungen zwischen China und Zentralasien, die Ent- } \\
\text { wicklung der SCO u. a. } \\
\text { Website: <http://www.xjass.com/zys/> (nur chinesisch) }\end{array}$ \\
\hline & $\begin{array}{l}\text { Xinjiang University, In- } \\
\text { stitute for Central Asian } \\
\text { Studies (ICAS) }\end{array}$ & $\begin{array}{l}\text { Das ICAS an der Xinjiang Universität wurde offiziell } 1987 \text { als Insti- } \\
\text { tut für zentralasiatische Kulturstudien gegründet. Doch waren be- } \\
\text { reits seit } 1979 \text { diverse Forschungsgruppen eingerichtet worden, die } \\
\text { sich mit Zentralasien im weiteren Sinne beschäftigten; damals hatte } \\
\text { man beispielsweise eine Abteilung für Zentralasiatische Geschichte } \\
\text { gegründet. Das Spektrum der Forschung umfasst heute Geschichte, } \\
\text { Kultur, Politik, Wirtschaft und Sicherheitsfragen Zentralasiens. } \\
2012 \text { hat die Xinjiang University gemeinsam mit der Tsinhua Uni- } \\
\text { versity ein Forschungszentrum für Zentralasienforschung ins Leben } \\
\text { gerufen, das am ICAS angesiedelt ist. } \\
\text { Website: <http://zyyjy.xju.edu.cn/> (nur chinesisch) }\end{array}$ \\
\hline Lanzhou & $\begin{array}{l}\text { University of Lanzhou, } \\
\text { Institute for Central Asian } \\
\text { Studies (ICAS) }\end{array}$ & $\begin{array}{l}\text { Das } 1994 \text { gegründete ICAS an der Universität Lanzhou ist heute } \\
\text { eine der wichtigsten Forschungseinrichtungen zum Thema Zentral- } \\
\text { asien innerhalb des chinesischen Universitätssystems. Das Institut } \\
\text { behandelt in seiner Forschung ein breites Themenspektrum, u. a. } \\
\text { Politik, Sicherheitsfragen, Wirtschaft, Studien zu Ethnizität und } \\
\text { Religion, Geschichte und Kultur etc. Seit } 2014 \text { wird am ICAS } \\
\text { die wissenschaftliche Zeitschrift Central Asia Studies (informelle } \\
\text { Übersetzung des Titels, erscheint nur chinesisch, Anm. der Red.) } \\
\text { herausgegeben. } \\
\text { Website: <http://icas.lzu.edu.cn/> (nur chinesisch) }\end{array}$ \\
\hline Xi'an & $\begin{array}{l}\text { Shaanxi Normal University } \\
\text { (SNU), Institute for Central } \\
\text { Asian Studies (ICAS) }\end{array}$ & $\begin{array}{l}\text { Das ICAS an der SNU wurde } 2004 \text { gegründet und ist Bestand- } \\
\text { teil des Instituts für die Westliche Grenzregion Chinas, einer der } \\
\text { führenden an Universitäten angesiedelten Forschungsinstitutionen, } \\
\text { die sich mit Zentralasien beschäftigen. Am Institut wird über viele } \\
\text { Themengebiete gearbeitet; ein besonderer Schwerpunkt liegt auf den } \\
\text { Beziehungen zwischen Nordwestchina und seinen Nachbarländern. } \\
\text { Website: <http://nec.snnu.edu.cn/College_profile.asp?id=14> (nur } \\
\text { chinesisch) }\end{array}$ \\
\hline
\end{tabular}




\section{März - 22. April 2016}

\section{Kasachstan}

\begin{tabular}{|c|c|}
\hline 27.3.2016 & $\begin{array}{l}\text { Eine Maschine der kasachstanischen Fluglinie Bek Air aus Kysylorda muss mit einer Störung am vorderen Fahr- } \\
\text { werk in Astana notlanden. Passagiere kommen nicht zu Schaden. }\end{array}$ \\
\hline 28.3.2016 & $\begin{array}{l}\text { Der ehemalige Leiter des Staatskomitees für Information und Kommunikation, Bolat Bersebajew, wird wegen } \\
\text { des Verdachts der Veruntreuung von Haushaltsmitteln festgenommen. }\end{array}$ \\
\hline 30.3 .2016 & $\begin{array}{l}\text { Am Flughafen von Astana stirbt der Leiter der Landesvertretung der ADB, der chinesische Staatsbürger Yang } \\
\text { Zhinlin, an einem Herzinfarkt. }\end{array}$ \\
\hline 30.3 .2016 & $\begin{array}{l}\text { Ein Gericht in Almaty setzt die Strafe des im Januar } 2016 \text { verurteilen Bürgerrechtlers Serikschan Mambetalin } \\
\text { von zwei Jahren Haft auf ein Jahr Bewährung, die seines Mitangeklagten Jermek Narymbajew von drei Jah- } \\
\text { ren Haft auf zwei Jahre Bewährung herab. }\end{array}$ \\
\hline 30.3 .2016 & $\begin{array}{l}\text { Präsident Nursultan Nasarbajew trifft in Brüssel mit dem Präsidenten der Europäischen Kommission, Jean- } \\
\text { Claude Juncker, und EU-Ratspräsident Donald Tusk zu Gesprächen über das bilaterale Verhältnis und aktuelle } \\
\text { Aspekte der europäischen und internationalen Politik zusammen. }\end{array}$ \\
\hline 30.3 .2016 & $\begin{array}{l}\text { Nationalbankchef Danijar Akischew prognostiziert bei einem Wirtschaftsforum in Astana, dass die Inflation } \\
\text { im Jahr } 20168 \% \text { nicht überschreiten werde. }\end{array}$ \\
\hline 31.3 .2016 & $\begin{array}{l}\text { Präsident Nasarbajew trifft zu einem Staatsbesuch in Washington ein, wo er u. a. am Gipfel für nukleare Sicher- } \\
\text { heit teilnimmt. Zudem sind bilaterale Treffen, u. a. mit US-Außenminister John Kerry, vorgesehen. }\end{array}$ \\
\hline 1.4 .2016 & $\begin{array}{l}\text { Der ehemalige Bürgermeister der Stadt Ust-Kamenogorsk, Manarbek Sapargalijew, wird wegen Annahme von } \\
\text { Schmiergeldern zu drei Jahren Haft verurteilt. }\end{array}$ \\
\hline 1.4 .2016 & $\begin{array}{l}\text { Der kasachstanische Boxer Gennadij Golowkin wird von Präsident Nasarbajew zum offiziellen EXPO-2017- } \\
\text { Botschafter ernannt. }\end{array}$ \\
\hline 1.4 .2016 & $\begin{array}{l}\text { Die chinesischen Behörden überstellen zwei wegen Drogenhandels verurteilte kasachstanische Staatsbürge- } \\
\text { rinnen in ihre Heimat. }\end{array}$ \\
\hline 2.4.2016 & $\begin{array}{l}\text { Präsident Nasarbajew trifft zu seinem ersten offiziellen Staatsbesuch in der kubanischen Hauptstadt Havanna } \\
\text { ein, wo er u. a. von seinem Amtskollegen Raul Castro zu Gesprächen empfangen wird. }\end{array}$ \\
\hline 4.4 .2016 & $\begin{array}{l}\text { Im neuen The Richest Countries oft he World Ranking des US-amerikanischen Global Finance Magazine } \\
\text { erscheint Kasachstan auf Platz } 50 \text { und damit noch kurz vor Russland. }\end{array}$ \\
\hline 5.4 .2016 & $\begin{array}{l}\text { Nach Angaben des Organized Crime and Corruption Reporting Project (OCCRP) enthalten die geleakten } \\
\text { Panama Papers auch Informationen über Offshore-Konten von Präsident Nasarbajews Enkel Nurali, der im } \\
\text { März von seinem Amt als stellvertretender Bürgermeister von Astana zurückgetreten war. Präsident Nasarba- } \\
\text { jew hat sich zuletzt im März } 2016 \text { in einer Rede vor dem Parlament öffentlich gegen die Nutzung von Brief- } \\
\text { kastenfirmen ausgesprochen. }\end{array}$ \\
\hline 5.4 .2016 & $\begin{array}{l}\text { Tengrinews meldet, dass die kasachstanische Regierung das internationale Karachaganak-Konsortium im Streit } \\
\text { um die Verteilung der Gewinne aus dem Gasfeld auf die Zahlung von 1,6 Mrd. US-Dollar verklagt habe. Das } \\
\text { Karachaganak-Feld ist für } 45 \% \text { der Gasproduktion Kasachstans verantwortlich. }\end{array}$ \\
\hline 6.4 .2016 & $\begin{array}{l}\text { Präsident Nasarbajew ruft in Telefonaten mit seinen armenischen und aserbaidschanischen Amtskollegen, Sersch } \\
\text { Sargsjan und Ilham Alijew, beide Seiten angesichts der massiven Kämpfe in und um Nagorny-Karabach in den } \\
\text { vergangenen Tagen zur Deeskalation auf. Auch mit Russlands Präsident Wladimir Putin bespricht Nasarba- } \\
\text { jew telefonisch die Lage in der Region. }\end{array}$ \\
\hline 7.4.2016 & $\begin{array}{l}\text { Auf Vorschlag Kasachstans, das } 2016 \text { den EEU-Vorsitz innehat, wird der für den 8.4. im armenischen Jere- } \\
\text { wan geplante Gipfel der Organisation auf den 13.4. in Moskau verschoben. Grund sind die Kämpfe in } \\
\text { Nagorny-Karabach. }\end{array}$ \\
\hline 7.4 .2016 & $\begin{array}{l}\text { Der wegen Korruptionsverdacht unter Hausarrest gestellte ehemalige Vorsitzende des Journalistenverbandes, } \\
\text { Seitkasy Matajew, wird erneut wegen gefährlich hohen Blutdrucks in ein Krankenhaus in Almaty eingeliefert. }\end{array}$ \\
\hline 7.4 .2016 & $\begin{array}{l}\text { Die Kurzdoku "Karachaganak: Kinder im Tausch gegen Öl« erreicht beim Kurzfilmfestival über Menschen- } \\
\text { rechte in Caracas (Venezuela) den zweiten Platz. }\end{array}$ \\
\hline 8.4 .2016 & Außenminister Jerlan Idrissow reist zum GUS-Außenministertreffen nach Moskau. \\
\hline 8.4 .2016 & $\begin{array}{l}\text { Die offizielle Website des Kremls meldet, dass Präsident Nasarbajew erneut telefonisch mit seinem russischen } \\
\text { Amtskollegen Putin die Lage in Nagorny-Karabach besprochen hat. }\end{array}$ \\
\hline
\end{tabular}




\begin{tabular}{|c|c|}
\hline 11.4 .2016 & $\begin{array}{l}\text { Ein Gericht in der Stadt Kostanai verurteilt den ehemaligen Bürgermeister der Stadt, Achmedbek Achmet- } \\
\text { schanow, zu } 13 \text { Jahren Lagerhaft wegen der Annahme von Schmiergeldern in besonders großem Umfang. Sein } \\
\text { früherer Stellvertreter, Maksut Kalijew, wird zu vier Jahren Haft verurteilt. Drei in gleicher Sache angeklagte } \\
\text { Geschäftsmänner müssen für zehn, } 11 \text { und } 14 \text { Jahre hinter Gitter. }\end{array}$ \\
\hline 11.4.2016 & $\begin{array}{l}\text { Präsident Nasarbajew trifft zu einem Staatsbesuch in Teheran ein, wo er u. a. von seinem iranischen Amtskol- } \\
\text { legen Hasan Ruhani und dem geistlichen Führer des Landes, Ali Khamenei, zu Gesprächen über das bilate- } \\
\text { rale Verhältnis empfangen wird. Derweil unterzeichnen iranische und kasachstanische Geschäftsleute Wirt- } \\
\text { schaftsverträge im Umfang von insgesamt mehr als } 1 \text { Mrd. US-Dollar. }\end{array}$ \\
\hline 12.4 .2016 & $\begin{array}{l}\text { Tengrinews meldet, dass der Konkurs gegangene Betrieb zur Herstellung von Tablet-Computern und Flach- } \\
\text { bildschirmen in Aktau (Gebiet Mangistau) in Zukunft Ersatzteile für Solarbatterien liefern werde. }\end{array}$ \\
\hline 12.4 .2016 & In der Nähe von Astana finden Manöver der Armee zur Erprobung des Anti-Terrorkampfes statt. \\
\hline 12.4.2016 & $\begin{array}{l}\text { In den Gebieten Aktöbe und Kostanai werden mehrere Überlandstraßen wegen der Gefahr von Unterspülung } \\
\text { durch stark angeschwollene Flüsse gesperrt. }\end{array}$ \\
\hline 12.4 .2016 & $\begin{array}{l}\text { Präsident Nasarbajew trifft in Ankara zum 13. Gipfel der Organisation für Islamische Zusammenarbeit (OIC) } \\
\text { ein, wo er auch mit seinem türkischen Amtskollegen Recep Tayyip Erdogan und dem saudischen König Sal- } \\
\text { man Albdelaziz al-Saud Gespräche über die bilateralen Beziehungen führt. }\end{array}$ \\
\hline 13.4.2016 & $\begin{array}{l}\text { Der bisherige stellvertretende Gouverneur des Gebiets Pawlodar, Nurschan Aschimbajew, wird zum neuen Bür- } \\
\text { germeister der Stadt Pawlodar ernannt. }\end{array}$ \\
\hline 14.4 .2016 & $\begin{array}{l}\text { Aus dem Energieministerium wird gemeldet, dass der frühere Ressortchef Wladimir Schkolnik zum außeror- } \\
\text { dentlichen Berater von Präsident Nasarbajew ernannt wurde. }\end{array}$ \\
\hline 15.4.2016 & $\begin{array}{l}\text { Präsident Nasarbajew wird im Rahmen eines Arbeitsbesuches in Taschkent von seinem usbekischen Amtskol- } \\
\text { legen Islam Karimow zu Gesprächen über die bilaterale Zusammenarbeit empfangen. }\end{array}$ \\
\hline 15.4.2016 & $\begin{array}{l}\text { Premier Massimow lädt bei einem Treffen mit EU-Landwirtschaftskommissar Phil Hogan in Astana europäi- } \\
\text { sche Unternehmen zu Investitionen im kasachstanischen Landwirtschaftssektor ein. }\end{array}$ \\
\hline 18.4.2016 & $\begin{array}{l}\text { Der Direktor der Metrogesellschaft in Almaty, Aidyn Oraschanow, wird nach zahlreichen Beschwerden von } \\
\text { Mitarbeitern entlassen und eine Untersuchung angeordnet. Oraschanow wird vorgeworfen, seit } 2014 \text { mehr als } \\
250 \text { Mitarbeiter entlassen und durch "eigene Leuten« ersetzt zu haben. Bereits zwei seiner Vorgänger, Kuat Musa- } \\
\text { bekow und Murat Ukschebajew, waren wegen Vetternwirtschaft und Korruptionsvorwürfen entlassen worden. }\end{array}$ \\
\hline 19.4.2016 & $\begin{array}{l}\text { Ein Gericht in der Stadt Karaganda beschließt in Reaktion auf ein entsprechendes Ersuchen die Entlassung } \\
\text { des wegen Korruption verurteilten ehemaligen Bürgermeisters der Stadt, Meiram Smagulow, aus der Haft und } \\
\text { die Umwandlung in eine Bewährungsstrafe. Der ehemalige Gouverneur des Gebiets Karaganda, Bauyrschan } \\
\text { Abdischew, zieht ein ähnliches Ersuchen zurück und bleibt weiterhin in Haft. }\end{array}$ \\
\hline 20.4 .2016 & $\begin{array}{l}\text { Der stellvertretende Wirtschaftsminister, Kairbek Uskenbajew, schließt im Staatssender Chabar die Veräuße- } \\
\text { rung von landwirtschaftlichen Flächen an ausländische Investoren kategorisch aus. }\end{array}$ \\
\hline 21.4.2016 & $\begin{array}{l}\text { Der Wechselkurs des Tenge gegenüber dem US-Dollar erreicht mit 332,8:1 den höchsten Stand seit Beginn } \\
\text { des Jahres. }\end{array}$ \\
\hline 21.4.2016 & $\begin{array}{l}\text { Durch Unterschrift von Präsident Nasarbajew tritt ein Kinderschutzgesetz in Kraft, das u. a. die chemische } \\
\text { Kastration von pädophilen Straftätern erlaubt. }\end{array}$ \\
\hline 21.4.2016 & $\begin{array}{l}\text { Im Rahmen des in Astana stattfindenden Eurasischen Medienforums erklärt der ehemalige afghanische Präsi- } \\
\text { dent Hamid Karsai seine Hochachtung für Präsident Nasarbajews Vermittlungsbemühungen in seiner Heimat. }\end{array}$ \\
\hline 22.4.2016 & $\begin{array}{l}\text { Eine litauische Regierungsdelegation unter Leitung von Wirtschaftsminister Evaldas Gustas, trifft zu bilate- } \\
\text { ralen Gesprächen in Astana ein. }\end{array}$ \\
\hline 22.4.2016 & $\begin{array}{l}\text { Energieminister Kanat Bosumbajew äußert die Erwartung, dass die Erdölförderung in Kaschagan noch Ende } \\
2016 \text { aufgenommen werden kann. }\end{array}$ \\
\hline
\end{tabular}

\section{Kirgistan}

26.3.2016

Der Pressedienst von Präsident Almasbek Atambajew meldet den Abzug usbekischer Truppen aus dem umstrittenen Grenzabschnitt im Bezirk Aksy (Gebiet Dschalalabad) als Ergebnis der Gespräche der Kommandierenden der Grenztruppen beider Länder am Vortag. 


\begin{tabular}{|c|c|}
\hline 27.3.2016 & $\begin{array}{l}\text { In den Städten Osch, Karakol, Balykschy, Tokmok, Kemin, Majluu-Suu und einer Reihe ländlicher Siedlun- } \\
\text { gen finden Wahlen für die Kommunalparlamente statt. Aus Osch werden neun Fälle versuchten Stimmen- } \\
\text { kaufs gemeldet. }\end{array}$ \\
\hline 28.3 .2016 & $\begin{array}{l}\text { Dastan Sarygulow, Staatssekretär in der Amtszeit Kurmanbek Bakijews, wird wegen des Verdachts eines } \\
\text { Umsturzversuchs unter Hausarrest gestellt. In gleicher Sache waren zuvor bereits der ehemalige Gouverneur } \\
\text { von Dschalalabad, Bektur Asanow, und die beiden Politiker Kubanytschbek Kadyrow und Ernest Karybekow } \\
\text { in Untersuchungshaft genommen worden. }\end{array}$ \\
\hline 28.3 .2016 & $\begin{array}{l}\text { Aus dem Komitee für soziale Fragen des Parlaments wird bekannt, dass sich unter den illegal nach Syrien Aus- } \\
\text { gereisten bislang } 125 \text { Kinder, in der Mehrzahl unter zehn Jahren alt, befunden haben. }\end{array}$ \\
\hline 29.3 .2016 & $\begin{array}{l}\text { In einer gemeinsamen Aktion russischer und kirgisischer Sicherheitskräfte wurde in den letzten Tagen in Mos- } \\
\text { kau eine Gruppe von kirgisischen Staatsbürgern verhaftet, die sich das Vertrauen ihrer als Arbeitsmigranten in } \\
\text { Moskau lebenden Landsleute erschlichen und sie dann ausgeraubt haben sollen. }\end{array}$ \\
\hline 29.3 .2016 & $\begin{array}{l}\text { Die Regierung verurteilt die Verbreitung gefälschter Protokolle über die Abtretung des Berges Ungar-Too (Gebiet } \\
\text { Dschalalabad) an der Grenze zu Usbekistan an das Nachbarland über die sozialen Netzwerke. Es handele sich } \\
\text { um eine Provokation des Oppositionellen Asimbek Beknasarow. }\end{array}$ \\
\hline 30.3 .2016 & $\begin{array}{l}\text { Nach Angaben des Direktors der Abteilung Tourismus im Ministerium für Kultur, Information und Touris- } \\
\text { mus, Asamat Dschamankulow, ist bis } 2020 \text { eine jährliche Steigerung der Zahl ausländischer Touristen um } \\
20 \% \text { und des Anteils des Tourismus am BIP auf nicht weniger als } 7 \% \text { (derzeit } 4,5 \% \text { ) angestrebt. Die Masse } \\
\text { der Touristen kommt bislang aus der GUS, für die Zukunft setzt man verstärkt auf China. }\end{array}$ \\
\hline 31.3 .2016 & $\begin{array}{l}\text { Bundesaußenminister Frank-Walter Steinmeier trifft während seiner Zentralasienreise in Bischkek mit Prä- } \\
\text { sident Atambajew und seinem Amtskollegen Erlan Abdyldajew zusammen, Themen der Gespräche sind u. a. } \\
\text { Extremismus und Islamismus. }\end{array}$ \\
\hline 31.3 .2016 & $\begin{array}{l}\text { Bei einer Sitzung des Parlaments wird bekannt, dass in den vergangenen vier Jahren } 62 \text { Wehrdienstleistende } \\
\text { ums Leben gekommen sind, } 70 \% \text { davon durch Selbstmord. }\end{array}$ \\
\hline 1.4 .2016 & $\begin{array}{l}\text { Der Pressedienst des Verteidigungsministeriums meldet, dass der Chef des Generalstabs der russischen Streit- } \\
\text { kräfte, General Walerij Gerasimow, dem Vorsitzenden des kirgisischen Staatskomitees für Verteidigung, Gene- } \\
\text { ralmajor Marat Kenisarijew, bei einem Treffen in Moskau russische Unterstützung bei der Modernisierung der } \\
\text { kirgisischen Armee zugesagt hat. }\end{array}$ \\
\hline 4.4 .2016 & $\begin{array}{l}\text { Der Pressesekretär von Premier Temir Sarijew wendet sich gegen in Medien und Gesellschaft aufgetauchte } \\
\text { Gerüchte, dass der Rücktritt der Regierung unmittelbar bevorstehe. }\end{array}$ \\
\hline 4.4 .2016 & $\begin{array}{l}\text { Mit der Unterschrift Präsident Atambajews unter die entsprechenden Erlasse werden die Jahrestage der Ereig- } \\
\text { nisse von Aksy (17.3.2002), der Tulpenrevolution (24.3.2005) und des Sturzes Kurmanbek Bakijews (7.4.2010) } \\
\text { als »bedeutendste Ereignisse der jüngsten Geschichte Kirgistans auf dem Weg zu Freiheit, Demokratie und } \\
\text { Gerechtigkeit« zu staatlichen Feiertagen erklärt. }\end{array}$ \\
\hline 4.4 .2016 & $\begin{array}{l}\text { Die Regierung beschließt in geschlossener Sitzung vier noch aus der Sowjetzeit von Usbekistan beanspruchte } \\
\text { und langfristig von usbekischen Staatsbürgern gepachtete Sanatorien am Issyk-Kul zu beschlagnahmen. }\end{array}$ \\
\hline 4.4 .2016 & $\begin{array}{l}\text { Eine Delegation des United Nations Peacebuilding Fund (PBF) trifft zu einem mehrtägigen Besuch in Kir- } \\
\text { gistan ein, bei dem hochrangige Gespräche geführt und geförderte Projekte in den Gebieten Osch und Bat- } \\
\text { ken besucht werden sollen. }\end{array}$ \\
\hline 5.4 .2016 & $\begin{array}{l}\text { Nach Angaben des Pressedienstes der Regierung haben sich die ausländischen Direktinvestitionen aus Russ- } \\
\text { land } 2015 \text { verdoppelt, die aus Kasachstan gingen um } 15 \% \text {, die aus China um } 51 \% \text { zurück. }\end{array}$ \\
\hline 5.4 .2016 & $\begin{array}{l}\text { Nach den Worten von Premier Sarijew konnte Kirgistan } 2015 \text { mit 3,5\% das stärkste Wirtschaftswachstum } \\
\text { aller EEU-Staaten verzeichnen. }\end{array}$ \\
\hline 6.4 .2016 & $\begin{array}{l}\text { Fergananews.com meldet, dass die chinesische State Power Investment Corporation eine Absichtserklärung über } \\
\text { den Bau des Kasarmanskij Wasserkraftwerkes (Gebiet Dschalalabad) am Naryn abgegeben habe. }\end{array}$ \\
\hline 6.4 .2016 & $\begin{array}{l}\text { Präsident Atambajew lehnt die von Premier Sarijew gewünschte Entlassung von Transportminister Argynbek } \\
\text { Malabajew ab. Sarijew und Malabajew schieben sich gegenseitig die Verantwortung für die rechtlich nicht zwei- } \\
\text { felsfreie Vergabe der Ausschreibung für den Ausbau der } 104 \mathrm{~km} \text { langen Überlandstraßenabschnitts Balykschy- } \\
\text { Tscholpon-Ata-Korumdu (Gebiet Issyk-Kul) im Wert von } 100 \text { Mio. US-Dollar zu. }\end{array}$ \\
\hline 6.4 .2016 & $\begin{array}{l}\text { Die russische Nachrichtenagentur RIA Nowosti meldet, dass Russland in Kirgistan (und Tadschikistan) gemein- } \\
\text { same regionale Luftabwehrsysteme stationieren wird. Der Vertrag über ein gemeinsames System der GUS-Staa- } \\
\text { ten war bereits } 1995 \text { unterzeichnet worden. }\end{array}$ \\
\hline
\end{tabular}




\begin{tabular}{|c|c|}
\hline 7.4.2016 & $\begin{array}{l}\text { Die thailändische Prinzessin Maha Chakri Sirindhorn erörtert während ihres ersten Kirgistan-Besuches mit } \\
\text { Präsident Atambajew Fragen der bilateralen Beziehungen. }\end{array}$ \\
\hline 8.4 .2016 & $\begin{array}{l}\text { Der Vorsitzende der Staatsduma der RF, Sergej Naryschkin, trifft während seines zweitägigen Arbeitsbesu- } \\
\text { ches in Bischkek mit Präsident Atambajew, Premier Sarijew und Parlamentssprecher Asylbek Dscheenbekow } \\
\text { zusammen. Beide Seiten betonen die große Bedeutung und den positiven Stand der bilateralen Beziehungen. }\end{array}$ \\
\hline 9.4 .2016 & $\begin{array}{l}\text { Eine auf Initiative des Abgeordneten der Partei Kyrgyzstan, Kanat Isajew, gebildete Kommission von Abge- } \\
\text { ordneten zur Untersuchung der Vorgänge bei der Entscheidung über die Straßenbau-Ausschreibung am Issyk- } \\
\text { Kul stellt Gesetzesverstöße bei der Vergabe fest und schlägt ein Misstrauensvotum gegen Premier Sarijew vor. }\end{array}$ \\
\hline 11.4 .2016 & $\begin{array}{l}\text { Premierminister Sarijew tritt in Reaktion auf die Korruptionsvorwürfe zurück, weist aber alle Anschuldigun- } \\
\text { gen zurück, er sei Opfer einer Kampagne von Lügen und Intrigen. Präsident Atambajew nimmt den Rücktritt } \\
\text { der Regierung an, die Minister bleiben aber bis zur Wahl ihrer Nachfolger im Amt. }\end{array}$ \\
\hline 11.4 .2016 & $\begin{array}{l}\text { Präsident Atambajew unterzeichnet das Gesetz über die Ratifizierung der Vereinbarung zwischen Kirgistan } \\
\text { und Indien über militärische Zusammenarbeit vom 12.7.2015. }\end{array}$ \\
\hline 12.4 .2016 & $\begin{array}{l}\text { Mitglieder der Menschenrechtsorganisation Bir Duino (Eine Welt) demonstrieren vor dem Parlamentsgebäude } \\
\text { in Bischkek gegen die zur Annahme anstehende Änderung des Gesetzes über nichtkommerzielle Organisatio- } \\
\text { nen, weil es demokratische Prinzipien verletze. }\end{array}$ \\
\hline 12.4 .2016 & $\begin{array}{l}\text { Der amtierende Außenminister Abdyldajew nimmt an der Sitzung des Rates der Außenminister der OIC-Staa- } \\
\text { ten in Istanbul teil. Am Rande der Veranstaltung führt Abdyldajew bilaterale Gespräche mit seinen Amtskol- } \\
\text { legen aus Bahrain, Afghanistan und Iran, Sheikh Khalid bin Ahmed bin Mohammed Al Khalifah, Salahud- } \\
\text { din Rabbani und Mohammad Javad Zarif. }\end{array}$ \\
\hline 12.4 .2016 & $\begin{array}{l}\text { Nach Angaben des nationalen Komitees für Statistik ist das BIP im ersten Quartal } 2016 \text { im Vergleich zum Vor- } \\
\text { jahreszeitraum um 4,9\% auf } 79 \text { Mrd. Som (ca. 1,15 Mrd. US-Dollar) gesunken. }\end{array}$ \\
\hline 13.4 .2016 & $\begin{array}{l}\text { Der Russisch-Kirgisische Entwicklungsfonds unterstützt kleine und mittlere Unternehmen in Kirgistan mit } \\
\text { einer Gesamtsumme von 2,2 Mrd. Som ( } 30,5 \text { Mio. US-Dollar), wie kirgisische Medien berichten. Gleichzei- } \\
\text { tig wurden zwei staatlichen Banken, der Ayyl Bank sowie der RSK Bank, zusätzlich } 700 \text { Mio. Som (9,7 Mio. } \\
\text { US-Dollar) zur Verfügung gestellt, so eigene Angaben des Entwicklungsfonds. }\end{array}$ \\
\hline 13.4 .2016 & Kirgistan wird auf dem EEU-Gipfel in Moskau vom amtierenden Vize-Premier Oleg Pankratow vertreten. \\
\hline 13.4.2016 & $\begin{array}{l}\text { Der ehemalige Gouverneur von Osch und bisherige stellvertretende Leiter des Apparats des Präsidenten, Soo- } \\
\text { ronbai Dscheenbekow, von der Sozialdemokratischen Partei wird von Vertretern aller Parteien zum neuen } \\
\text { Premierminister gewählt. In seiner Regierung gibt es nur wenige Veränderungen, u. a. wird Samir Aidarow } \\
\text { neuer Transportminister. Der bisherige Sprecher des Parlaments und ältere Bruder des neuen Premier, Asyl- } \\
\text { bek Dscheenbekow, tritt zurück. }\end{array}$ \\
\hline 14.4 .2016 & $\begin{array}{l}\text { Der Energieminister der VAE, Suhail Mohamed Faraj Al Mazrouei, und der kirgisische Wirtschaftsminister } \\
\text { Arsybek Kojoschew erklären auf dem Kyrgyzstan-United Arab Emirates Economic Forum in Bischkek ihr Inte- } \\
\text { resse an einer verstärkten ökonomischen Zusammenarbeit. }\end{array}$ \\
\hline 14.4 .2016 & Das Parlament wählt wider Erwarten keinen neuen Sprecher, weil nicht genügend Abgeordnete anwesend sind. \\
\hline 14.4 .2016 & $\begin{array}{l}\text { Das Parlament billigt mit 73:45 Stimmen die noch vom Vorgänger-Parlament initiierte umstrittene Änderung } \\
\text { des Gesetzes über nichtkommerzielle Organisationen, allerdings ohne die umstrittene Formulierung, die Orga- } \\
\text { nisationen, die sich mit Politik befassen und Geld aus dem Ausland erhalten, zu ausländischen Agenten erklärte. }\end{array}$ \\
\hline 18.4 .2016 & $\begin{array}{l}\text { Präsident Atambajew unterzeichnet eine Änderung des Strafvollzugskodexes, mit der die von anderen Häft- } \\
\text { lingen getrennte Unterbringung wegen extremistischer und terroristischer Vergehen verurteilter Gefangener } \\
\text { festgelegt wird. }\end{array}$ \\
\hline 19.4.2016 & $\begin{array}{l}\text { Finanzminister Adylbek Kasymalijew und der Leiter der Abteilung Zentralasien beim Direktorat für interna- } \\
\text { tionale Entwicklung und Zusammenarbeit bei der Europäischen Kommission, Jobst von Kirchmann, unter- } \\
\text { zeichnen einen Vertrag, mit dem die EU Kirgistan } 36 \text { Mio. Euro für Reformen im Bildungssektor und } 13 \text { Mio. } \\
\text { Euro zur Unterstützung der nächsten Wahlen zur Verfügung stellt. }\end{array}$ \\
\hline 20.4 .2016 & $\begin{array}{l}\text { Zum Abschluss seines zweitägigen Bischkek-Besuches erklärt der EU-Sonderbeauftragte für Zentralasien, Peter } \\
\text { Burian, dass Kirgistan der wichtige Partner der EU in Zentralasien sei. }\end{array}$ \\
\hline 20.4 .2016 & $\begin{array}{l}\text { Die Wahl des neuen Parlamentssprechers endet mit einem Ergebnis von } 51: 51 \text { für die beiden Kandidaten Bakyt } \\
\text { Torobajew (Onuguu-Progress) und Kanat Isajew (Kyrgyzstan) und macht einen neuen Wahlgang mit neuen } \\
\text { Kandidaten erforderlich. }\end{array}$ \\
\hline 20.4 .2016 & Premierminister Dscheenbekow ernennt Asamat Sulajmanow zum neuen Chef des staatlichen Zolldienstes. \\
\hline
\end{tabular}




\begin{tabular}{|l|l|}
\hline 21.4.2016 & $\begin{array}{l}\text { Der UN-Menschenrechtsrat fordert Kirgistan auf, den usbekischen Menschenrechtler Asimschan Askarow, der } \\
\text { 2010 in einem umstrittenen Urteil wegen Anstiftung zu ethnischem Hass im Zusammenhang mit den Ereig- } \\
\text { nissen in Osch zu einer lebenslänglichen Freiheitsstrafe verurteilt worden war, freizulassen und ihm eine Ent- } \\
\text { schädigung zu zahlen, da das Urteil nicht rechtmäßig gewesen sei. }\end{array}$ \\
\hline 21.4.2016 & $\begin{array}{l}\text { Ein Regierungssprecher teilt vor dem Parlament mit, dass die Bildung einer speziell ausgebildeten Grenztruppe } \\
\text { für den Einsatz an der Grenze zu Usbekistan beschlossen wurde. }\end{array}$ \\
\hline
\end{tabular}

\section{Tadschikistan}

\begin{tabular}{|c|c|}
\hline 28.3.2016 & $\begin{array}{l}\text { Ein Sprecher der Grenztruppen teilt mit, dass zwei am 25.3. von afghanischen Schmugglern entführte Bewoh- } \\
\text { ner des Bezirkes Schuroobod (Gebiet Chatlon) befreit und auf dem Weg nach Hause seien. }\end{array}$ \\
\hline 28.3 .2016 & $\begin{array}{l}\text { Tadschikische und kirgisische Grenzschützer verhindern die Eskalation eines Konfliktes zwischen Bewohnern } \\
\text { des tadschikischen Dorfes Tschorkuch (Gebiet Sogd) und des kirgisischen Aksaj (Gebiet Batken) über die Nut- } \\
\text { zung von Kies aus dem Fluss Karawschin. }\end{array}$ \\
\hline 29.3.2016 & $\begin{array}{l}\text { Im Rahmen einer ganzen Reihe von Umbesetzungen entlässt Präsident Emomali Rachmon den Direktor der } \\
\text { nationalen Informationsagentur Tadschikistans Chowar, Kosimi Sarobiddin, und ernennt ihn zum Botschaf- } \\
\text { ter in Saudi-Arabien. }\end{array}$ \\
\hline 29.3.2016 & $\begin{array}{l}\text { Der Bürgermeister von Duschanbe kündigt an, dass in Zukunft zur Sicherheit der Betenden und Aufrechter- } \\
\text { haltung der Ordnung alle Moscheen der Stadt mit Videokameras überwacht werden. }\end{array}$ \\
\hline 30.3 .2016 & $\begin{array}{l}\text { Nach Angaben des tadschikischen Dienstes von RFE/RL haben bislang nur } 312 \text { der insgesamt } 1.600 \text { Rechts- } \\
\text { anwälte des Landes die seit einer Gesetzesänderung im November } 2015 \text { für ihre Zulassung bis } 28.3 \text { abzule- } \\
\text { gende Prüfung über juristische, politische und kulturelle Fragen bestanden. Das Parlament verabschiedet am } \\
\text { gleichen Tag eine Gesetzesänderung, die die Sicherheitsorgane verpflichtet, Verhafteten einen Rechtsanwalt zu } \\
\text { stellen und Angehörige zu informieren. }\end{array}$ \\
\hline 30.3 .2016 & $\begin{array}{l}\text { Das Unterhaus des Parlamentes beschließt die umstrittene Veränderung des Mediengesetzes in geänderter Fas- } \\
\text { sung. Statt der kritisierten Regelung, dass das auch für die Registrierung von Massenmedien zuständige Kultur- } \\
\text { ministerium über ein dreimonatiges Verbot entscheiden darf, soll die Entscheidung nun bei den Gerichten liegen. }\end{array}$ \\
\hline 30.3 .2016 & $\begin{array}{l}\text { Gerichte in Duschanbe verurteilen zwei Arbeitsmigranten, die auf dem Weg nach Syrien im November } 2015 \\
\text { in der Türkei verhaftet und in ihre Heimat ausgeliefert worden waren, zu je sieben Jahren Freiheitsentzug. }\end{array}$ \\
\hline 30.3 .2016 & $\begin{array}{l}\text { Der Landesdirektor der ADB für Tadschikistan, C.C.Yu, erklärt auf einer Pressekonferenz in Duschanbe, dass } \\
\text { die Forschungsergebnisse seines Instituts ein Fortbestehen der Wirtschaftskrise in diesem Jahr anzeigen und } \\
\text { schlägt die Erarbeitung eines neuen Modells der ökonomischen Entwicklung, orientiert auf Wasserenergie, } \\
\text { Öko-Tourismus und Landwirtschaft vor. Die Führung des Landes reagiert mit der Erklärung, dass Präsident } \\
\text { Rachmon diese Ideen längst gehabt habe. }\end{array}$ \\
\hline 30.3 .2016 & Tadschikistan und Nicaragua nehmen in New York diplomatische Beziehungen auf. \\
\hline 31.3 .2016 & $\begin{array}{l}\text { Aus einer Fernsehsendung des Kanals Safina geht hervor, dass zwei Bewohner des Bezirkes Dschlikul unter } \\
\text { dem Verdacht der Vorbereitung eines Anschlages auf das Gebäude der Gebietsverwaltung und der Miliz von } \\
\text { Chatlon festgenommen wurden. }\end{array}$ \\
\hline 1.4 .2016 & $\begin{array}{l}\text { Bundesaußenminister Frank-Walter Steinmeier führt in Duschanbe Gespräche mit Präsident Rachmon und } \\
\text { Außenminister Sirojiddin Aslow über Fragen der globalen und regionalen Sicherheit und Wirtschaft. }\end{array}$ \\
\hline 4.4 .2016 & $\begin{array}{l}\text { Tadschikistan ist mit Rang } 153 \text { (von 183) und einem Pro-Kopf-BIP (PPP) von ca. } 2.800 \text { US-Dollar der ärmste } \\
\text { GUS-Staat im Rating The Richest Countries in the World des amerikanischen Global Finance Magazine. }\end{array}$ \\
\hline 4.4 .2016 & $\begin{array}{l}\text { Ein Gericht in Duschanbe verurteilt mehrere Verwandte des im Herbst } 2015 \text { aufständischen ehemaligen stell- } \\
\text { vertretenden Verteidigungsministers Abduhalim Nazarzoda zu mehrjährigen Haftstrafen. }\end{array}$ \\
\hline 5.4 .2016 & $\begin{array}{l}\text { Der tadschikische Schriftstellerverband präsentiert ein Buch mit dem Titel „Der Führer ist der Stolz der Nation« } \\
\text { der Öffentlichkeit, in dem Wissenschaftler, Schriftsteller und Politiker Präsident Rachmon lobpreisen. }\end{array}$ \\
\hline 6.4 .2016 & $\begin{array}{l}\text { Das Unterhaus verabschiedet eine Änderung des Luftverkehrskodexes, nach der unbemannte Flugkörper } \\
\text { (Drohnen, Quadrikopter) nur mit Erlaubnis der Regierung über tadschikischem Staatsgebiet fliegen dürfen. }\end{array}$ \\
\hline 6.4 .2016 & $\begin{array}{l}\text { In einer Rede vor dem Unterhaus des Parlamentes teilt Nematullo Hikmatullosoda, Minister für ökonomi- } \\
\text { sche Entwicklung und Handel, mit, dass Deutschland 2016/17 für Gesundheitsvorsorge, regionale Entwick- } \\
\text { lung, den Privatsektor der Wirtschaft und Bau bezahlbaren Wohnraums 54,5 Mio. Euro zur Verfügung stellt. }\end{array}$ \\
\hline
\end{tabular}




\begin{tabular}{|c|c|}
\hline 6.4 .2016 & $\begin{array}{l}\text { Die russische Nachrichtenagentur RIA Nowosti meldet, dass Russland in Tadschikistan (und Kirgistan) gemein- } \\
\text { same regionale Luftabwehrsysteme stationieren wird. Der Vertrag über ein gemeinsames System der GUS-Staa- } \\
\text { ten war bereits } 1995 \text { unterzeichnet worden. }\end{array}$ \\
\hline 7.4 .2016 & $\begin{array}{l}\text { In Duschanbe versammeln sich mehr als sechzig weibliche Führungskräfte aus allen zentralasiatischen Repu- } \\
\text { bliken zu einem von USAID finanzierten Runden Tisch zum Thema Förderung weiblichen Unternehmertums. }\end{array}$ \\
\hline 7.4 .2016 & $\begin{array}{l}\text { Die erste direkte Luftverkehrsverbindung zwischen Tadschikistan und Pakistan nimmt auf der Strecke } \\
\text { Duschanbe-Lahore den Betrieb auf. }\end{array}$ \\
\hline 8.4 .2016 & $\begin{array}{l}\text { Bei einem Treffen von Finanzminister Abdusalom Kurbonijon mit Vertretern der EBRD unter Führung von } \\
\text { Richard Jones werden Perspektiven der Zusammenarbeit, insbesondere die weitere Finanzierung der Instand- } \\
\text { setzung der Straße von Duschanbe zur usbekischen Grenze, erörtert. }\end{array}$ \\
\hline 8.4 .2016 & $\begin{array}{l}\text { Am Rande der Sitzung der Außenminister der GUS-Staaten in Moskau treffen Außenminister Aslow und sein } \\
\text { russischer Amtskollege Sergej Lawrow zu Gesprächen über Fragen des bilateralen Verhältnisses zusammen. }\end{array}$ \\
\hline 8.4 .2016 & $\begin{array}{l}\text { Mufti Saidmukarram Abdukodirsoda, Vorsitzender der Rates der Ulama der islamischen Zentren, beschuldigt } \\
\text { in seiner Freitagspredigt in der zentralen Moschee von Duschanbe islamische Parteien, Streit und Spaltung in } \\
\text { muslimische Länder zu bringen und als destabilisierender Faktor zu wirken. }\end{array}$ \\
\hline 8.4 .2016 & $\begin{array}{l}\text { Ein Gericht im Bezirk Wachsch (Gebiet Chatlon) verurteilt einen 17jährigen wegen des Versuchs, von Mos- } \\
\text { kau aus nach Syrien auszureisen, zu einer Freiheitsstrafe von sieben Jahren. }\end{array}$ \\
\hline 8.4 .2016 & $\begin{array}{l}\text { HRW weist in einer Erklärung im Vorfeld der mit der Erstellung des Universal Periodic Review durch den } \\
\text { UN-Menschenrechtsrat verbundenen Tadschikistanbesuche internationaler Delegationen auf die sich im letz- } \\
\text { ten Jahr stark verschlechterte Situation der Menschenrechte und der Pressefreiheit sowie des Umgangs mit poli- } \\
\text { tische Opposition hin. }\end{array}$ \\
\hline 8.4 .2016 & $\begin{array}{l}\text { Am Abend werden zwei tadschikische Arbeitsmigranten in der Moskauer Metro von einem betrunkenen Fahr- } \\
\text { gast erst beschimpft und dann einer mit zwei Schüssen aus einer Gaspistole aus kurzer Entfernung am Kopf } \\
\text { schwer verletzt. }\end{array}$ \\
\hline 10.4 .2016 & $\begin{array}{l}\text { Bei Nachwahlen für das Unterhaus des Parlamentes in zwei Wahlkreisen (in Duschanbe und im Gebiet Sogd) } \\
\text { wird in beiden Fällen der Kandidat der regierenden Volksdemokratischen Partei Tadschikistans gewählt. }\end{array}$ \\
\hline 13.4 .2016 & $\begin{array}{l}\text { Dem bekannten tadschikischen Kinoregisseur Barsu Abdurassakow wird auf dem Flughafen Moskau Domo- } \\
\text { djedowo die Einreise nach Russland verweigert. Abdurassakow, der in Bischkek lebt und arbeitet, wird nach } \\
\text { Duschanbe deportiert. }\end{array}$ \\
\hline 14.4 .2016 & $\begin{array}{l}\text { Der Stadtrat von Duschanbe nominiert Präsidententochter Osoda Rachmon als Kandidatin für die für den } \\
\text { 29.5. wegen einer Umbesetzung notwendig gewordene Nachwahl in den Senat. }\end{array}$ \\
\hline 14.4 .2016 & $\begin{array}{l}\text { In Duschanbe werden vier Männer wegen Zugehörigkeit zu den Salafiten zu Freiheitsstrafen von drei bis } 16 \\
\text { Jahren verurteilt. }\end{array}$ \\
\hline 15.4 .2016 & $\begin{array}{l}\text { Die untere Kammer des Parlamentes ratifiziert den im Februar } 2016 \text { unterzeichneten Vertrag über den Ausbau } \\
\text { des Heizkraftwerkes Schurob zwischen der tadschikischen Regierung und der deutschen Hos Powertech Inter- } \\
\text { national SDN BND. In der ersten Projektphase wird die Firma } 400 \text { Mio. US-Dollar investieren. }\end{array}$ \\
\hline 15.4 .2016 & $\begin{array}{l}\text { Das Parlament verabschiedet eine Änderung des Gesetzes über Feiertage mit der der 16. November zum Tag } \\
\text { des Präsidenten erklärt wird, der aber nicht arbeitsfrei sein soll. }\end{array}$ \\
\hline 15.4 .2016 & $\begin{array}{l}\text { Der tadschikische Dienst von RFE/RL berichtet, dass seit einigen Tagen wegen Qualitätsbedenken Einfuhr- } \\
\text { verbote für iranische Produkte gelten. }\end{array}$ \\
\hline 16.4 .2016 & $\begin{array}{l}\text { In Guliston (bisher Kairakkum, Gebiet Sogd) endet die erste Sitzung des im Juni } 2015 \text { beschlossenen gemein- } \\
\text { samen Kollegiums der Außenministerien Tadschikistans und Kirgistans mit der Unterzeichnung von Doku- } \\
\text { menten über eine verstärkte Zusammenarbeit im Kampf gegen Extremismus und Terrorismus. }\end{array}$ \\
\hline 16.4 .2016 & $\begin{array}{l}\text { Tadschikistan taucht erstmals im jährlichen Report über Religionsfreiheit des U.S. State Department als Staat } \\
\text { auf, in dem die Religionsfreiheit verletzt wird. }\end{array}$ \\
\hline 18.4 .2016 & $\begin{array}{l}\text { Nach Angaben der US-Botschaft in Duschanbe hat der Kommandierende des United States Special Operati- } \\
\text { ons Command Central (SOCCENT), Generalmajor Darsie D. Rogers, Tadschikistan und Usbekistan besucht } \\
\text { und dort Gespräche über Sicherheit und Stabilität in der Region geführt. }\end{array}$ \\
\hline 18.4 .2016 & $\begin{array}{l}\text { Auf dem Truppenübungsgelände Magob beginnt unter Teilnahme von Verteidigungsminister Scherali Mirso, } \\
\text { CSTO-Generalsekretär Nikolaj Bordjuscha und dem Chef des gemeinsamen Stabes des CSTO, Generaloberst } \\
\text { Anatolij Sidorow, das erste gemeinsame Manöver der militärischen Geheimdienste der CSTO-Staaten. }\end{array}$ \\
\hline
\end{tabular}




\begin{tabular}{|c|c|}
\hline 19.4 .2016 & $\begin{array}{l}\text { Ein Gericht in Guliston (bisher Kairakkum, Gebiet Sogd) verurteilt die 28jährige Rawschana Mirchodscha- } \\
\text { jewa wegen des Versuchs, am syrischen Bürgerkrieg teilzunehmen, zu einer 12jährigen Freiheitsstrafe. Ihr Mann } \\
\text { war schon } 2015 \text { wegen des gleichen Delikts verurteilt worden. }\end{array}$ \\
\hline 19.4 .2016 & $\begin{array}{l}\text { Der Sprecher der unteren Kammer des Parlamentes, Schukurdschon Suchurow, nimmt am ersten Treffen der } \\
\text { Sprecher der Parlamente Eurasiens in Moskau teil. Am Rande des dreitägigen Treffens tritt Suchurow mit sei- } \\
\text { nem iranischen Amtskollegen Ali Laridschani zusammen. }\end{array}$ \\
\hline 20.4 .2016 & $\begin{array}{l}\text { In der neuesten Rangliste der Pressefreiheit von Reporter ohne Grenzen ist Tadschikistan gegenüber dem Vor- } \\
\text { jahr um } 34 \text { Plätze abgestürzt und liegt nun auf Rang } 150 \text { von } 180 \text { gelisteten Staaten. }\end{array}$ \\
\hline 20.4 .2016 & $\begin{array}{l}\text { Bei einer Schlägerei zwischen tadschikischen und kaukasischen Migranten in Moskau kommt ein 24jähriger } \\
\text { Tadschike ums Leben. }\end{array}$ \\
\hline 20.4 .2016 & $\begin{array}{l}\text { Außenminister Aslow wird am Rande seines Aufenthaltes in New York zur Teilnahme an der 30. Sondersitzung } \\
\text { der Generalversammlung der UN zum Drogenproblem vom Vertreter des UN-Generalsekretärs für soziale und } \\
\text { wirtschaftliche Fragen, Wu Hongbo, empfangen. }\end{array}$ \\
\hline 20.4 .2016 & $\begin{array}{l}\text { Nach heftigen Regenfällen kommt es im Gebiet Chatlon zu Überschwemmungen und Erdrutschen, bei denen } \\
\text { allein im Dorf Bahoriston } 100 \text { Häuser zerstört werden. }\end{array}$ \\
\hline 21.4 .2016 & $\begin{array}{l}\text { Premier Kohir Rasulsoda empfängt in Duschanbe den EU-Sonderbeauftragten für Zentralasien, Peter Burian, } \\
\text { zu Gesprächen über das bilaterale Verhältnis. }\end{array}$ \\
\hline 21.4 .2016 & $\begin{array}{l}\text { Der unter dem Pseudonym Anas bekannte Anuscherwon Asimow aus Nurek, der Hunderte von Tadschiken } \\
\text { für den IS angeworben haben soll, ist in Syrien umgekommen, meldet der tadschikische Dienst von RFE/RL } \\
\text { unter Berufung auf tadschikische Regierungsstellen. }\end{array}$ \\
\hline 21.4 .2016 & $\begin{array}{l}\text { Der Pressedienst von TALCO (Tajik Aluminum Company), dem größten Industrieunternehmen Tadschikistans, } \\
\text { meldet, dass während des Besuches des Präsidenten der schweizerischen Handelsgesellschaft Glencore Inter- } \\
\text { national AG, Ivan Glasenberg, ein Übereinkommen über die Lieferung von } 200.000 \text { t Rohaluminium an } \\
\text { TALCO erreicht wurde. }\end{array}$ \\
\hline 22.4 .2016 & $\begin{array}{l}\text { Nach Angaben der Agentur für Statistik betrug der Außenhandelsumsatz Tadschikistans im ersten Quartal } \\
2016 \text { 874,2 Mio. US-Dollar und war damit 9,2\% niedriger als im Vergleichszeitraum des Vorjahres, dabei sank } \\
\text { der Export um 8,2\% und der Import um 9,9\%. }\end{array}$ \\
\hline 22.4 .2016 & $\begin{array}{l}\text { Der tadschikische Dienst von RFE/RL meldet unter Berufung auf Menschenrechtsorganisationen, dass } 2015 \\
\text { mindestens zehn tadschikische Journalisten in den USA oder Europa um politisches Asyl nachgesucht haben. }\end{array}$ \\
\hline
\end{tabular}

\section{Turkmenistan}

\begin{tabular}{|c|c|}
\hline 26.3.2016 & $\begin{array}{l}\text { Gesundheitsminister Nurmuchammed Amannesepow wird von Präsident Gurbanguly Berdymuchammedow } \\
\text { während einer erweiterten Kabinettssitzung wegen nicht zufrieden stellender Arbeitsergebnisse scharf gerügt. }\end{array}$ \\
\hline 27.3.2016 & $\begin{array}{l}\text { Nachdem Präsident Berdymuchammedow Verteidigungsminister Jailym Berdijew dazu aufgefordert hatte, umge- } \\
\text { hend und -fassend die Kampfstärke der Armee zu überprüfen, beginnen groß angelegte, landesweite Manöver } \\
\text { unter Einbeziehung aller Teilstreitkräfte. }\end{array}$ \\
\hline 1.4 .2016 & $\begin{array}{l}\text { In Istanbul wurden bei einer Razzia der türkischen Polizei gegen einen Drogenschmugglerring } 21 \text { turkmeni- } \\
\text { sche Staatsangehörige festgenommen, meldet Radio Azatlyk. }\end{array}$ \\
\hline 2.4 .2016 & $\begin{array}{l}\text { Der Verantwortliche für Auswärtiges und Außenhandel des Gebiets Astrachan der RF, Denis Afanasjew, erklärt } \\
\text { nach einer Delegationsreise nach Aschchabad, bei der vor allem die Tourismusförderung im Mittelpunkt stand, } \\
\text { dass er bis Ende } 2016 \text { mit Visaerleichterungen für russische Touristen nach Turkmenistan rechnet. }\end{array}$ \\
\hline 5.4 .2016 & Ein neues Gesetz tritt in Kraft, das die Reklame u. a. für Glücksspiel untersagt. \\
\hline 6.4 .2016 & $\begin{array}{l}\text { Präsident Berdymuchammedow zeigt sich bei einem Treffen des Nationalen Sicherheitsrates zufrieden mit dem } \\
\text { Ergebnis der zahlreichen im Land abgehaltenen Manöver. }\end{array}$ \\
\hline 6.4 .2016 & $\begin{array}{l}\text { Es wird ein Gesetz verabschiedet, wonach vor einer Hochzeit für beide Ehepartner zukünftig ein HIV-Test ver- } \\
\text { pflichtend wird. Zudem werden HIV-Tests für der Drogenabhängigkeit Verdächtige, Ausländer, die ein Arbeits- } \\
\text { visum beantragen, Häftlinge und Blutspender vorgeschrieben. }\end{array}$ \\
\hline 7.4.2016 & $\begin{array}{l}\text { Der Monat für Gesundheit und Glück beginnt in Aschchabad und in den Gebietshauptstädten mit großen } \\
\text { Fahrraddemonstrationen. }\end{array}$ \\
\hline
\end{tabular}




\begin{tabular}{|c|c|}
\hline 8.4 .2016 & $\begin{array}{l}\text { Der Regionalchef der ADB für Zentral- und Westasien, Sean O'Sullivan, gibt bekannt, dass sein Institut Turk- } \\
\text { menistan einen Kredit in Höhe von } 1 \text { Mrd. US-Dollar für den Ausbau von Eisenbahntrassen und der Energie- } \\
\text { versorgungsinfrastruktur zur Verfügung stellt. }\end{array}$ \\
\hline 8.4 .2016 & Außenminister Raschid Meredow reist zum Außenministertreffen der GUS nach Moskau. \\
\hline 8.4 .2016 & $\begin{array}{l}\text { Mehrere staatliche Unternehmen aus Pakistan, Indien und Afghanistan sowie Turkmengaz unterzeichnen ein } \\
\text { Investitionsabkommen über den Bau der TAPI-Pipeline. }\end{array}$ \\
\hline 9.4 .2016 & $\begin{array}{l}\text { Präsident Berdymuchammedow entlässt während einer erweiterten Kabinettssitzung Wirtschafts- und Ent- } \\
\text { wicklungsminister Jaldesch Scheripow, Handelsminister Bajar Abajew und den Chef der Steuerbehörde, Schat- } \\
\text { lyk Chummedow, wegen schwerer Versäumnisse in ihrer Amtsführung. Deren Nachfolger werden Batyr Basa- } \\
\text { row, Dowran Orasmyradow und Saparberdy Gundogdyjew. Auch die für Kultur zuständige Vize-Premier- } \\
\text { ministerin, Maisa Jasmuchammedowa, muss ihren Platz räumen, Nachfolgerin wird Gulschat Mammedowa. }\end{array}$ \\
\hline 9.4 .2016 & $\begin{array}{l}\text { Präsident Berdymuchammedow äußert sich vor dem Kabinett unzufrieden mit der langsamen wirtschaftli- } \\
\text { chen Entwicklung und konstatiert, dass die zahlreichen Sozialleistungen an die Bevölkerung ihre Berechti- } \\
\text { gung verloren hätten. }\end{array}$ \\
\hline 13.4 .2016 & $\begin{array}{l}\text { Der Gouverneur des Gebiets Astrachan der RF, Aleksandr Schilkin, wird von Präsident Berdymuchammedow } \\
\text { zu Gesprächen über das bilaterale Verhältnis empfangen. }\end{array}$ \\
\hline 14.4 .2016 & $\begin{array}{l}\text { Der bekannte russische Blogger und Traveller Ilja Warlamow erhält als Reaktion auf einen kritischen Beitrag } \\
\text { über die Besorgnis erregende (Menschenrechts-) Lage in Turkmenistan einen Tweet mit einer Todesdrohung, } \\
\text { der nach Vermutungen des oppositionellen Portals Chronika Turkmenistana vom Bürgermeister der turkme- } \\
\text { nischen Stadt Balkanabat (Gebiet Balkan), Emin Aschyrow, stammen könnte. }\end{array}$ \\
\hline 16.4 .2016 & $\begin{array}{l}\text { er für die Präsidentenadministration zuständige Vize-Premier, Schamuchammet Durdylyjew, wird von Prä- } \\
\text { dent Berdymuchammedow im Rahmen einer erweiterten Kabinettssitzung scharf wegen Nachlässigkeiten in } \\
\text { r Amtsführung gerügt. }\end{array}$ \\
\hline 16.4 .2016 & $\begin{array}{l}\text { Das oppositionelle Nachrichtenportal Gündogar.org meldet, dass nach am 13.4. in Kraft getretenen Geset- } \\
\text { zesänderungen die Strafe für die Vermietung von Wohnungen ohne Registrierung um das Zehnfache ange- } \\
\text { hoben wurde. }\end{array}$ \\
\hline 16.4 .2016 & $\begin{array}{l}\text { In Aschchabad wird eine im Jahr } 1993 \text { mit Spenden von Gläubigen erbaute Moschee auf Anordnung der Behör- } \\
\text { den abgerissen, um Raum für den Bau neuer Wohnungen zu schaffen. }\end{array}$ \\
\hline 17.4 .2016 & $\begin{array}{l}\text { Präsident Berdymuchammedow fordert den Leiter der Agentur für Öl- und Gasförderung, Jagschygeldy Kaka- } \\
\text { jew, dazu auf, Schritte zur Förderung von Investitionen zur Ausbeutung dieser Rohstoffe im Küstenschelf des } \\
\text { Kaspischen Meeres zu unternehmen. }\end{array}$ \\
\hline 18.4 .2016 & $\begin{array}{l}\text { Die russische Nachrichtenagentur RIA Nowosti meldet, dass nach Unterschrift unter das entsprechende Gesetz } \\
\text { durch Präsident Berdymuchammedow ab dem 1.5. einige Exportbeschränkungen für turkmenische Waren auf- } \\
\text { gehoben bzw. gelockert werden. Genaueres wird nicht genannt. }\end{array}$ \\
\hline 18.4 .2016 & $\begin{array}{l}\text { Die oppositionelle Website Chronika Turkmenistana meldet, dass sich die Militäreinheiten im Gebiet Mary an } \\
\text { der Grenze zu Afghanistan seit Anfang April in erhöhter Gefechtsbereitschaft befinden. }\end{array}$ \\
\hline 20.4 .2016 & $\begin{array}{l}\text { Der Chef der Abteilung für Zentralasien des russischen Außenministeriums, Samir Kabulow, erklärt auf einer } \\
\text { Pressekonferenz, dass er angesichts der aktuellen kritischen Lage in Afghanistan keine Chancen für eine Fort- } \\
\text { setzung des TAPI-Projekts sehe. }\end{array}$ \\
\hline 22.4.2016 & $\begin{array}{l}\text { In Aschchabad wird das neue Buch von Präsident Berdymuchammedow über die Achalteke-Pferderasse offi- } \\
\text { ziell der Öffentlichkeit vorgestellt. }\end{array}$ \\
\hline
\end{tabular}

\section{Usbekistan}

\begin{tabular}{|l|l|}
\hline 28.3.2016 & $\begin{array}{l}\text { Die Menschenrechtsorganisationen Association for Human Rights in Central Asia (AHRCA) und Internatio- } \\
\text { nal Partnership for Human Rights (IPHR) drücken ihre Besorgnis darüber aus, dass der am 9.1.2016 in Tasch- } \\
\text { kent unter Extremismusverdacht verhaftete russische Staatsbürger Bachtijor Chudajberdijew gefoltert werde. }\end{array}$ \\
\hline 28.3.2016 & $\begin{array}{l}\text { Der Bürgermeister von Taschkent, Rachmonbek Usmanow, teilt vor der Presse mit, dass der Straßenbahn- } \\
\text { verkehr in Taschkent eingestellt wird. Das 87,8 km lange veraltete Schienennetz solle zugunsten des Autover- } \\
\text { kehrs demontiert werden. }\end{array}$ \\
\hline
\end{tabular}




\begin{tabular}{|c|c|}
\hline 29.3 .2016 & $\begin{array}{l}\text { Erst jetzt wird gemeldet, dass die russische Duma eine Vereinbarung vom Dezember } 2014 \text { über die Regelung } \\
\text { gegenseitiger Schulden aus den Jahren } 1992 / 93 \text { ratifiziert hat. Russland erlässt Usbekistan } 864,3 \text { Mio. US- } \\
\text { Dollar, Taschkent verzichtet auf Ansprüche aus dem Diamantenfonds und Währungsreserven auf Konten der } \\
\text { Außenhandelsbank der UdSSR. }\end{array}$ \\
\hline 29.3 .2016 & $\begin{array}{l}\text { Der Anfang der 2000er Jahre in Usbekistan bekannte Popsänger Abdulasis Karim erhält nach nur zehn Mona- } \\
\text { ten die türkische Staatsbürgerschaft. Er hatte Usbekistan im Mai } 2015 \text { endgültig verlassen, aber bereits seit } \\
2005 \text { Probleme und Auftrittsverbote wegen seiner kritischen Liedtexte. }\end{array}$ \\
\hline 30.3 .2016 & $\begin{array}{l}\text { Bundesaußenminister Frank-Walter Steinmeier trifft während seines eintägigen Besuches in Taschkent mit Prä- } \\
\text { sident Islam Karimow und seinem Amtskollegen Abdulasis Kamilow zusammen. Steinmeier betont das deut- } \\
\text { sche Interesse an einem Dialog, mahnt aber auch die Einhaltung der Menschenrechte an. }\end{array}$ \\
\hline 30.3 .2016 & $\begin{array}{l}\text { Im niederländischen Tiel stirbt 58jährig nach schwerer Krankheit der Gründer der für Demokratie und Men- } \\
\text { schenrechte eintretenden, exiloppositionellen Website Zamondosh, Risochodscha Obidow. Der Dichter war } \\
\text { in Folge der Ereignisse von Andischan ins Exil gegangen und als politischer Flüchtling anerkannt. Die Redak- } \\
\text { tion teilt die Einstellung der Website mit. }\end{array}$ \\
\hline 30.3 .2016 & $\begin{array}{l}\text { Die ABD senkt ihre Prognose für das BIP-Wachstum Usbekistans von } 7 \text { auf } 6,9 \% \text {. Die Regierung geht von } \\
7,8 \% \text { aus. }\end{array}$ \\
\hline 1.4 .2016 & $\begin{array}{l}\text { Mit Monatsbeginn steigen die Preise im öffentlichen Personennahverkehr Taschkents ohne vorherige Ankün- } \\
\text { digung um ca. } 20 \% \text {. }\end{array}$ \\
\hline 1.4 .2016 & $\begin{array}{l}\text { Mehr als } 150 \text { Bewohner des Dorfes Bulungur arik (Gebiet Samarkand) protestieren vor dem Gebäude der } \\
\text { Dorfversammlung gegen die Demontage von Gasleitungen, die sie selber auf eigene Kosten zu ihren Häusern } \\
\text { gelegt hatten. }\end{array}$ \\
\hline 2.4 .2016 & $\begin{array}{l}\text { Die Nachrichtenagentur Regnum meldet unter Berufung auf das Staatskomitee für Privatisierung, Demono- } \\
\text { polisierung und Wettbewerbsentwicklung, dass an der Taschkenter Börse staatliche Aktiva von } 59 \text { Gesellschaf- } \\
\text { ten und Banken zu einem Startpreis von ca. } 915 \text { Mio. US-Dollar zum Verkauf stehen, darunter Produzenten } \\
\text { von Mineraldünger, Öl- und Gasausrüster u. a.. }\end{array}$ \\
\hline 2.4 .2016 & $\begin{array}{l}\text { Eine südkoreanische Delegation unter Führung von Arbeitsminister Li Gi Kwon besucht Samarkand und dort } \\
\text { von Korea geförderte Einrichtungen. }\end{array}$ \\
\hline 4.4 .2016 & $\begin{array}{l}\text { Im Gebiet Buchara hat die mit einer Leistung von 1,2 MV größte mobile Sonnenenergiestation in der GUS } \\
\text { den Betrieb aufgenommen, meldet gazeta.uz. }\end{array}$ \\
\hline 4.4 .2016 & $\begin{array}{l}\text { Der usbekische Dienst von RFE/RL meldet, dass der bekannte Menschenrechtler Dmitrij Tichonow aus Angren } \\
\text { (Gebiet Taschkent), der Usbekistan im Februar verlassen hatte, in Deutschland politisches Asyl beantragt habe. }\end{array}$ \\
\hline 5.4 .2016 & $\begin{array}{l}\text { Der Parlamentarische Staatssekretär im britischen Außenministerium, Tobias Elwood, trifft zu Gesprächen mit } \\
\text { Außenminister Kamilow, Vertretern des Ministeriums für Außenhandelsbeziehungen u. a. in Taschkent ein. }\end{array}$ \\
\hline 5.4 .2016 & $\begin{array}{l}\text { Norma.uz meldet, dass die staatliche Kommission zur Kontrolle von Narkotika die Liste der verbotenen Dro- } \\
\text { gen von } 220 \text { auf } 289 \text { erheblich verlängert hat. In der Vergangenheit wurden auch Touristen verhaftet, wenn sie } \\
\text { gelistete Medikamente bei sich hatten. }\end{array}$ \\
\hline 6.4 .2016 & $\begin{array}{l}\text { Durch einen im abendlichen Hauptprogramm des staatlichen Fernsehens gezeigten Dokumentarfilm wird } \\
\text { bekannt, dass der usbekische Staatsbürger Scharifdschon Asrorow von einem Militärgericht wegen Spionage } \\
\text { für Tadschikistan zu } 16 \text { Jahren Freiheitsentzug verurteilt wurde. }\end{array}$ \\
\hline 8.4 .2016 & $\begin{array}{l}\text { Am Rande des Treffens der Außenminister der GUS-Staaten in Moskau trifft Außenminister Kamilow zu bila- } \\
\text { teralen Gesprächen mit seinem russischen Amtskollegen Sergej Lawrow zusammen. }\end{array}$ \\
\hline 8.4 .2016 & $\begin{array}{l}\text { In Taschkent findet ein reguläres Treffen der Anti-Terror-Struktur der SCO (RATS) statt, bei dem u. a. eine } \\
\text { Veränderung der rechtlichen Grundlagen des gemeinsamen Anti-Terror-Kampfes diskutiert wird. }\end{array}$ \\
\hline 8.4 .2016 & $\begin{array}{l}\text { Nach Angaben des Steuerkomitees wurden seit Anfang } 2016 \text { bei gezielten Aktionen gegen illegale Taxifahrer } \\
\text { ca. 7.500 Personen überführt, die insgesamt ca. 6,5 Mrd. Som (2,2 Mio. US-Dollar) Strafe zahlen mussten. }\end{array}$ \\
\hline 8.4 .2016 & $\begin{array}{l}\text { Der usbekische Dienst von RFE/RL berichtet, dass Mitarbeiter des Komitees für Staatssicherheit und der } \\
\text { Miliz usbekische Arbeitsmigranten in Russland und der Türkei mit telefonischen Drohungen zur Rückkehr } \\
\text { zu bewegen versuchen. }\end{array}$ \\
\hline 8.4 .2016 & $\begin{array}{l}\text { Air Cargo World meldet, dass Südkorea und Usbekistan eine Vereinbarung über den Gütertransport nach Iran } \\
\text { über das usbekische Luftverkehrskreuz Nawoi unterzeichnet haben. }\end{array}$ \\
\hline
\end{tabular}




\begin{tabular}{|c|c|}
\hline 9.4 .2016 & $\begin{array}{l}\text { Außenminister Kamilow empfängt in Taschkent U.S. Deputy Assistant Secretary for Central Asia Daniel } \\
\text { Rosenblum zu Gesprächen über Stand und Perspektiven der bilateralen Zusammenarbeit. }\end{array}$ \\
\hline 9.4 .2016 & $\begin{array}{l}\text { Der usbekische Dienst von RFE/RL meldet unter Berufung auf die Menschenrechtlerin Nadjeschda Atajaewa, } \\
\text { dass der langjährige persönliche Buchhalter von Präsidententochter Gulnara Karimowa, Nurmuchammad Sady- } \\
\text { kow, vor kurzem zu einer 18jährigen Freiheitsstrafe verurteilt worden sei. Er war vor drei Jahren wegen illega- } \\
\text { ler finanzieller Machenschaften verhaftet worden. }\end{array}$ \\
\hline 11.4 .2016 & $\begin{array}{l}\text { Auf Anordnungen von Präsident Karimow wird eine spezielle Außenhandelsgesellschaft für den Export von } \\
\text { frischem und verarbeitetem Obst und Gemüse gegründet und der staatliche Aufkauf von Obst und Gemüse } \\
\text { eingeführt. Die Regierung beschließt die Aufhebung der seit 1.9.2015 geltenden Exportbeschränkungen für } \\
\text { Obst und Gemüse ab 1.7.2016. }\end{array}$ \\
\hline 11.4 .2016 & $\begin{array}{l}\text { Eine Delegation der International Trade Union Confederation unter Leitung ihrer Generalsekretärin Sharan } \\
\text { Burrow besucht Usbekistan. }\end{array}$ \\
\hline 12.4 .2016 & $\begin{array}{l}\text { Das am Vortag von Präsident Karimow unterzeichnete Gesetz über Parlamentskontrolle tritt in Kraft. Objekte der } \\
\text { Kontrolle sind die Staatsorgane und die dort tätigen Personen bei Erfüllung ihrer verfassungsmäßigen Aufgaben. }\end{array}$ \\
\hline 12.4 .2016 & $\begin{array}{l}\text { Der usbekische Dienst von RFE/RL meldet, dass Ende letzter Woche Familienangehörige des in einem umstrit- } \\
\text { tenen Urteil wegen angeblicher Verbindungen zum IS verurteilten Aramais Awakjan und seines Freundes Fur- } \\
\text { kat Dschurajew in der US-Botschaft in Taschkent von U.S. Deputy Assistant Secretary for Central Asia Rosen- } \\
\text { blum empfangen wurden. }\end{array}$ \\
\hline 13.4 .2016 & $\begin{array}{l}\text { In Taschkent findet ein zweitägiges Treffen der Sekretäre der Sicherheitsräte der SCO-Staaten statt, auf dem } \\
\text { Fragen der regionalen und internationalen Sicherheit erörtert werden. Präsident Karimow hebt in seiner Begrü- } \\
\text { ßungsrede Notwendigkeit und Effektivität der SCO hervor. }\end{array}$ \\
\hline 13.4 .2016 & $\begin{array}{l}\text { Innenminister Adham Ahmatbojew und der chinesischen Minister für öffentliche Sicherheit, Guo Sheng- } \\
\text { kun, unterzeichnen in Taschkent ein Protokoll über den Ausbau der Zusammenarbeit in den Jahren 2016-17. }\end{array}$ \\
\hline $14 . .4 .2016$ & $\begin{array}{l}\text { Der usbekische Dienst von RFE/RL berichtet, dass mehrere in den letzten sechs Monaten in Taschkent und } \\
\text { den Gebieten Taschkent und Fergana unter dem Verdacht von Kontakten zum IS Festgenommene unter der } \\
\text { Bedingung, geheim mit den Strafverfolgungsbehörden zusammenzuarbeiten, freigelassen wurden. }\end{array}$ \\
\hline 15.4 .2016 & $\begin{array}{l}\text { Der kasachstanische Präsident Nursultan Nasarbajew wird in Taschkent von Präsident Karimow zu Gesprä- } \\
\text { chen über Fragen der bilateralen Zusammenarbeit und der internationalen Sicherheit empfangen. }\end{array}$ \\
\hline 15.4 .2016 & $\begin{array}{l}\text { In Taschkent wird das neue Abkommen mit UNICEF für die Jahre 2016-20 im Umfang von } 33 \text { Mio. US- } \\
\text { Dollar unterzeichnet, Ziel ist die Verbesserung der Situation von Frauen und Kindern. }\end{array}$ \\
\hline 16.4 .2016 & $\begin{array}{l}\text { Usbekische Medien melden für das erste Quartal } 2016 \text { ein BIP-Wachstum von 7,5\%. Entsprechend dem staat- } \\
\text { lichen Beschäftigungsprogramm seien in den ersten drei Monaten des Jahres } 2016 \text { mehr als } 165.000 \text { neue Stel- } \\
\text { len geschaffen worden. }\end{array}$ \\
\hline 16.4 .2016 & $\begin{array}{l}\text { Zum wiederholten Male zeigt das staatliche Fernsehen eine Sendung über die »Dschihaddisten« Awakjan und } \\
\text { Dschurajew und beschuldigt das Urteil kritisierende Menschenrechtler und westliche Medien, für ihre »Pro- } \\
\text { vokationen« bezahlt zu werden. }\end{array}$ \\
\hline 18.4 .2016 & $\begin{array}{l}\text { In Nawoi wird der 69jährige Schuchrat Nursatow vorzeitig aus der Haft entlassen. Er hatte } 1991 \text { als Deputier- } \\
\text { ter des Obersten Sowjets mit heftiger Kritik an Präsident Karimow Berühmtheit erlangt. } 2012 \text { war er wegen } \\
\text { Wirtschaftsverbrechen zu sieben Jahren Haft verurteilt worden. }\end{array}$ \\
\hline 19.4 .2016 & $\begin{array}{l}\text { Uzbekistan Airways teilt mit, dass die Gesellschaft ab 29.4. den regulären Frachtverkehr von Nawoi nach Tehe- } \\
\text { ran aufnimmt. }\end{array}$ \\
\hline 19.4 .2016 & $\begin{array}{l}\text { Laut RIA Nowosti investiert Usbekistan 2016/2017 } 145 \text { Mio. US-Dollar in die Schaffung der Infrastruktur } \\
\text { des e-governments. }\end{array}$ \\
\hline 19.4 .2016 & $\begin{array}{l}\text { In Anwesenheit des Präsidenten der russischen LUKoil, Wagit Alekperow, von Premierminister Schawkat Mir- } \\
\text { sijojew und des Gouverneurs des Gebiets Buchara, Muchiddin Esanow, wird feierlich der Grundstein für den } \\
\text { Gas verarbeitenden Komplex in Kandym (Gebiet Buchara) gelegt. }\end{array}$ \\
\hline 21.4 .2016 & $\begin{array}{l}\text { AI beschuldigt in einem neuen Report Russland entgegen internationalen Verträgen Hunderte Usbeken aus- } \\
\text { geliefert zu haben, obwohl ihnen in der Heimat Folter drohte. }\end{array}$ \\
\hline 21.4 .2016 & $\begin{array}{l}\text { Die schwedische Telekommunikationsgesellschaft TeliaSonera ändert ihren Namen in Telia Company, um } \\
\text { von ihrer Verwicklung in einen Korruptionsskandal mit Präsidententochter Gulnara Karimowa abzulenken. }\end{array}$ \\
\hline
\end{tabular}




\begin{tabular}{|l|l|}
\hline 21.4.2016 & $\begin{array}{l}\text { In zwei getrennten Gerichtsverhandlungen in Angren werden die Verfahren gegen den inzwischen im Aus- } \\
\text { land lebenden Menschenrechtler Dmitrij Tichonow wegen Hooliganismus und unerlaubten Foto- und Film- } \\
\text { aufnahmen eingestellt. }\end{array}$ \\
\hline 22.4.2016 & $\begin{array}{l}\text { Bloomberg News meldet, dass die US-Staatsanwaltschaft in einem Brief an einen Bundesrichter von Manhattan } \\
\text { erstmals ausdrücklich den Namen von Präsidententochter Gulnara Karimowa im Zusammenhang mit Ermitt- } \\
\text { lungen und Gerichtsurteilen wegen Korruption und Geldwäsche genannt hat. Nachdem Karimowa und ihre } \\
\text { Geschäftspartner ein Urteil, das sie zur Zahlung von Schadensersatz in Höhe von 550 Mio. US-Dollar verur- } \\
\text { teilte, ignoriert haben, wird in dem Brief die Pfändung von Konten beantragt. }\end{array}$ \\
\hline
\end{tabular}

Sie können die gesamte Chronik seit 2008 auch auf <http://www.laender-analysen.de/zentralasien/> unter dem Link »Chronik«lesen.

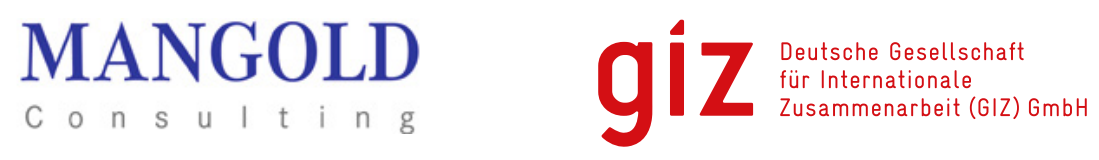

\footnotetext{
Herausgeber: Forschungsstelle Osteuropa an der Universität Bremen und Deutsche Gesellschaft für Osteuropakunde

Die Meinungen, die in den Zentralasien-Analysen geäußert werden, geben ausschließlich die Auffassung der Autoren wieder. Abdruck und sonstige publizistische Nutzung sind nach Rücksprache mit der Redaktion gestattet. Verantwortliche Redakteurin: Beate Eschment

Redaktionsassistenz: Henryk Alff

Satz: Matthias Neumann

Zentralasien-Analysen-Layout: Matthias Neumann, nach einen Konzept von Cengiz Kibaroglu, mit einer Grafik von Sebastian Klüsener Alle Ausgaben der Zentralasien-Analysen sind mit Themen- und Autorenindex archiviert unter www.laender-analysen.de Die Zentralasien-Analysen werden im Rahmen der Datenbank World Affairs Online (WAO) ausgewertet und sind im Portal IREON www.ireon-portal.de recherchierbar. ISSN 1866-2110 @ 2016 by Deutsche Gesellschaft für Osteuropakunde e.V. und Forschungsstelle Osteuropa, Bremen Deutsche Gesellschaft für Osteuropakunde e.V. • Schaperstraße 30 • 10719 Berlin • Telefon: +49 3021478412 • Telefax: +4930 21478414 e-mail: Zentralasien-Analysen@dgo-online.org •Internet-Adresse: www.laender-analysen.de/zentralasien
} 


\section{Kostenlose E-Mail-Dienste der Forschungsstelle Osteuropa und ihrer Partner auf www.laender-analysen.de}

Die Länder-Analysen bieten regelmäßig im kostenlosen Abonnement kompetente Einschätzungen aktueller politischer, wirtschaftlicher, sozialer und kultureller Entwicklungen in Ostmitteleuropa und der GUS. Sie machen das Wissen, über das die wissenschaftliche Forschung in reichem Maße verfügt, für Politik, Wirtschaft, Medien und die interessierte Öffentlichkeit verfügbar. Autoren sind internationale Fachwissenschaftler und Experten.

Die einzelnen Länder-Analysen werden von der Forschungsstelle Osteuropa an der Universität Bremen und der Deutschen Gesellschaft für Osteuropakunde jeweils mit unterschiedlichen Partnern und Sponsoren herausgegeben. Die Redaktionen der Länder-Analysen bestehen aus Wissenschaftlern mit langjähriger Forschungserfahrung.

Die Länder-Analysen bieten regelmäßig Kurzanalysen zu aktuellen Themen, ergänzt um Grafiken und Tabellen sowie Dokumentationen. Zusätzlich gibt es eine Chronik aktueller Ereignisse. Alle Länder-Analysen sind auch mit Archiv und Indizes online verfügbar unter $<$ www.laender-analysen.de $>$.

\section{Belarus-Analysen}

Erscheinungsweise: zweimonatlich

Abonnement unter: <http://www.laender-analysen.de/belarus/>

\section{Caucasus Analytical Digest}

In englischer Sprache. Erscheinungsweise: monatlich

Abonnement unter: <http://www.css.ethz.ch/en/publications/cad.html>

\section{Polen-Analysen}

Erscheinungsweise: zweimal monatlich

Abonnement unter: <http://www.deutsches-polen-institut.de/newsletter/polen-analysen/>

\section{Russland-Analysen}

Erscheinungsweise: zweiwöchentlich

Abonnement unter: <http://www.laender-analysen.de/russland/>

\section{Russian Analytical Digest}

In englischer Sprache. Erscheinungsweise: zweimal monatlich

Abonnement unter: <http://www.css.ethz.ch/en/publications/rad.html>

\section{Ukraine-Analysen}

Erscheinungsweise: zweimal monatlich

Abonnement unter: <http://www.laender-analysen.de/ukraine/>

\section{Zentralasien-Analysen}

Erscheinungsweise: monatlich

Abonnement unter: <http://www.laender-analysen.de/zentralasien/>

\section{Bibliographische Dienste}

Die Bibliographien informieren über englisch- und deutschsprachige Neuerscheinungen zu Belarus, Russland, Ukraine sowie zu den zentralasiatischen und kaukasischen Staaten. Erfasst werden jeweils die Themenbereiche Politik, Außenpolitik, Wirtschaft und Soziales.

Erscheinungsweise: viermal jährlich

Abonnement unter: <http://www.laender-analysen.de/bibliographies/belarus.php $>,<$ http://www.laender-analysen. de/bibliographies/russia.php>, <http://www.laender-analysen.de/bibliographies/ukraine.php >, <http://www.laenderanalysen.de/bibliographies/caucasus_ca.php> 